\title{
AtCGL160 recruits chloroplast coupling factor 1
}

2

3 Bennet Reiter ${ }^{1}$, Lea Rosenhammer ${ }^{1}$, Giada Marino ${ }^{1}$, Stefan Geimer ${ }^{2}$, Dario Leister ${ }^{1}$, 4 Thilo Rühle $1, \S$

5

6 1Plant Molecular Biology Faculty of Biology I, Ludwig-Maximilians-Universität Munich, 7 D-82152 Planegg-Martinsried, Germany (B.R., L.R., G.M., D.L., T.R.)

8 2Zellbiologie/Elektronenmikroskopie NW I/B1, Universität Bayreuth, 95447 Bayreuth,

9 Germany

$\S$ To whom correspondence should be addressed. E-mail: thilo.ruehle@biologie.uni12 muenchen.de

14 Running title: AtCGL160 links $\mathrm{CF}_{1}$ to a CFo assembly module 


\section{Abstract}

ATP synthases couple the generation of chemical energy to a transmembrane electrochemical potential. Like ATP synthases in bacteria and mitochondria, chloroplast ATP synthases consist of a membrane-spanning $\left(\mathrm{CF}_{\mathrm{O}}\right)$ and a soluble coupling factor $\left(\mathrm{CF}_{1}\right)$. Accessory factors facilitate subunit production and orchestrate the assembly of the functional $\mathrm{CF}_{1}-\mathrm{CF}_{\mathrm{O}}$ complex. It was previously shown that the accessory factor CGL160 promotes the formation of plant $\mathrm{CF}_{\mathrm{O}}$ and performs a similar function in the assembly of its c-ring to that of the distantly related bacterial Atp1/Uncl protein. In this study, we show that the $\mathrm{N}$-terminal portion of CGL160 (AtCGL160N), which is specific to the green lineage, is required for late steps in $\mathrm{CF}_{1}-\mathrm{CF}_{\mathrm{O}}$ assembly in Arabidopsis thaliana. In plants that lacked this stroma-exposed domain, photosynthesis was impaired, and amounts of $\mathrm{CF}_{1}-\mathrm{CF}_{\mathrm{O}}$ were reduced to about $65 \%$ of the wild-type level. Loss of AtCGL160N did not perturb c-ring formation, but led to a 10-fold increase in the numbers of $\mathrm{CF}_{1}$ sub-complexes in the stroma relative to the wild type and the $\mathrm{CF}_{1}$ assembly mutant atcgld11-1. Co-immunoprecipitation and protein crosslinking assays revealed an association of AtCGL160 with $\mathrm{CF}_{1}$ subunits. Yeast two-hybrid assays localized the interaction to a stretch of AtCGL160N that binds to the thylakoid-proximal domain of $\mathrm{CF}_{1-} \beta$ that includes the conserved DELSEED motif. We therefore propose that AtCGL160 has acquired an additional function in the recruitment of soluble $\mathrm{CF}_{1}$ to a membrane-integral $\mathrm{CF}_{\mathrm{O}}$ subcomplex, which is critical for the modulation of $\mathrm{CF}_{1}-\mathrm{CF}_{\mathrm{O}}$ activity and photosynthesis in chloroplasts.

Keywords: chloroplast, photosynthesis, ATP synthase, thylakoid complex, assembly, $\mathrm{CF}_{1-}$ $\mathrm{CF}_{\mathrm{O}}$, Arabidopsis 


\section{Introduction}

F-type ATP synthases, which utilize chemiosmotic membrane potentials to generate ATP, are central actors in the energy metabolism of bacteria, mitochondria and chloroplasts. These biological nanomotors share a largely conserved structure, consisting of a soluble $F_{1}$ and a membrane-bound $\mathrm{F}_{\mathrm{O}}$ moiety. Bacterial and chloroplast ATP synthases $\left(\mathrm{CF}_{1}-\mathrm{CF}_{0}\right)$ are closely related with respect to size and subunit composition (Groth and Pohl, 2001; Vollmar et al., 2009; Hahn et al., 2018) and, in contrast to the multimeric mitochondrial ATP synthases, exist as monomers in thylakoid membranes (Daum et al., 2010). In the chloroplasts of higher plants, $\mathrm{CF}_{1}-\mathrm{CF}_{\mathrm{O}}$ complexes reside exclusively in stroma lamellae and grana-end membranes, because the $\sim 16-\mathrm{nm}$ stromal extension of $\mathrm{CF}_{1}$ prevents its incorporation into the tightly packed grana stacks (Daum et al., 2010).

During photophosphorylation, $\mathrm{CF}_{1}-\mathrm{CF}_{0}$ complexes couple the light-driven generation of the trans-thylakoid proton-motive force ( $p m f)$ to ADP phosphorylation. The membrane-embedded proteolipidic $\mathrm{c}_{14}$-ring, together with the non-covalently bound central stalk $\gamma \varepsilon$, form the motor unit, and drive rotary catalysis by $\mathrm{CF}_{1}$. The peripheral stator consists of the subunits $\mathrm{a}, \mathrm{b}$ and $b^{\prime}$, and is connected to the $(\alpha \beta)_{3}$ unit by the $\delta$ subunit, which acts as a flexible hinge between $\mathrm{CF}_{1}$ and $\mathrm{CF}_{\mathrm{O}}$ (Murphy et al., 2019). Protons are translocated from the luminal to the stromal side via two aqueous channels in the a subunit. During translocation, each proton enters the access channel and binds to a conserved glutamate residue in subunit $\mathrm{c}$. The $\mathrm{c}_{14}$ motor executes an almost complete rotation before releasing the proton into the stroma through the exit channel (Hahn et al., 2018). The counterclockwise rotation of the central stalk in the vicinity of the hexamer triggers alternating nucleotide-binding affinities in the $\beta$ subunits that ultimately drive ATP generation (reviewed in von Ballmoos et al., 2009; Junge and Nelson, 2015).

As a result of extensive organellar gene transfer during plant evolution, three $\mathrm{CF}_{1}-\mathrm{CF}_{\mathrm{O}}$ subunits $\left(b^{\prime}, \gamma, \delta\right)$ are encoded in the nuclear genome, while the remaining $\mathrm{CF}_{1}-\mathrm{CF}_{\circ}$ genes are organized into two plastid operons. Consequently, two different gene-expression systems must be tightly coordinated with the chloroplast protein import machinery for efficient $\mathrm{CF}_{1}-\mathrm{CF}_{\mathrm{O}}$ biogenesis. Several $\mathrm{CF}_{1}-\mathrm{CF}_{\mathrm{O}}$ auxiliary factors involved in plastid gene expression have been identified, including proteins involved in mRNA processing (AEF1), mRNA stabilization (PPR10, BFA2) and translation initiation (ATP4, TDA1) (Pfalz et al., 2009; Eberhard et al., 2011; Zoschke et al., 2012; Yap et al., 2015; Zhang et al., 2019). Moreover, $\mathrm{CF}_{1}-\mathrm{CF}_{\mathrm{O}}$ assembly factors ensure correct complex stoichiometry, and prevent the accumulation of dead-end products or harmful intermediates that could lead to wasteful ATP hydrolysis or pmf dissipation. 
As in the case of the bacterial assembly model, plastid $\mathrm{CF}_{1}-\mathrm{CF}_{\mathrm{O}}$ complexes are constructed from different intermediates or modules (reviewed in Rühle and Leister, 2015). CF $_{1}$ assembly was first examined using in-vitro reconstitution assays, and was shown to be initiated by $\alpha / \beta$ dimerization in a chaperone-assisted process (Chen and Jagendorf, 1994). CF 1 formation depends on CGLD11/BFA3, which is specific to green plants, interacts with the hydrophobic catalytic site of the $\beta$-subunit and may prevent aggregation or formation of unfavorable homodimers (Grahl et al., 2016; Zhang et al., 2016). Moreover, PAB (Mao et al., 2015) and BFA1 (Zhang et al., 2018) have been proposed to be required for efficient incorporation of the $\gamma$ subunit into $\mathrm{CF}_{1}$.

Less is known about $\mathrm{CF}_{\mathrm{O}}$ assembly, and only one accessory factor - CONSERVED ONLY IN THE GREEN LINEAGE 160 (CGL160) - has been identified so far (Rühle et al., 2014). Absence of CGL160 in the Arabidopsis thaliana mutant atcgl160-1 is associated with a significant reduction (70-90\%) in wild-type $\mathrm{CF}_{1}-\mathrm{CF}_{\mathrm{O}}$ levels, and $\mathrm{CF}_{\mathrm{O}} \mathrm{C}$ subunits accumulate as monomers. Moreover, split-ubiquitin assays have provided evidence that AtCGL160 interacts with $\mathrm{CF}_{\mathrm{O}-\mathrm{C}}$ and $\mathrm{CF}_{\mathrm{O}}-\mathrm{b}$. It was therefore concluded that AtCGL160 is required for efficient formation of the c-ring in chloroplasts and shares this function with its distantly related bacterial counterpart Atp1/Uncl (Suzuki et al., 2007; Ozaki et al., 2008). Furthermore, AtCGL160 was suggested to participate in $\mathrm{CF}_{1}$ assembly into the holo-complex, based on $\mathrm{CF}_{1}$ subcomplex comigration and crosslinking experiments using a putatively specific anti-AtCGL160 antibody (Fristedt et al., 2015).

In this study, the function of the N-terminal domain that is conserved in all CGL160 proteins from the green lineage was investigated in Arabidopsis. The results demonstrate that this domain (AtCGL160N) mediates the critical connection of $\mathrm{CF}_{1}$ to $\mathrm{CF}_{\mathrm{O}}$ assembly modules by interacting with subunit $\beta$. Thus, CGL160 emerges as a key auxiliary factor that not only promotes $\mathrm{CF}_{\mathrm{O}}$ formation, but is also involved in late $\mathrm{CF}_{1}-\mathrm{CF}_{\mathrm{O}}$ assembly steps.

\section{Results}

The N-terminal moiety of AtCGL160 is required for efficient photosynthesis and $\mathrm{CF}_{1-}$ CFo functionality

CGL160 was identified based on its coregulation with photosynthetic genes in ATTED-II (Obayashi et al., 2009) and its affiliation to the GreenCut suite of proteins (Merchant et al., 2007; Karpowicz et al., 2011). The C-terminal transmembrane segment of CGL160 ( 15 kDa) is distantly related to bacterial Atp1/Uncl (Rühle et al., 2014; Fristedt et al., 2015), whereas the larger $\mathrm{N}$-terminal portion of the protein sequence is only conserved in algae, bryophytes, and 
higher plants (Supplemental Fig. 1). This latter domain of $\sim 200$ amino acids (aa) in Arabidopsis thaliana (AtCGL160N) includes a predicted N-terminal chloroplast transit peptide (cTP) of 46 aa (Emanuelsson et al., 1999), and mass spectrometry has identified several phosphorylated peptides which are derived from positions 106-134 (Reiland et al., 2009; Reiland et al., 2011; Roitinger et al., 2015). Indeed, two conserved putative phosphorylation sites were found in a multiple sequence alignment of CGL160 homologs from species across the green lineage, which correspond to positions S111 and S126 in AtCGL160 (Fig. 1, Supplemental Fig. 1).

A

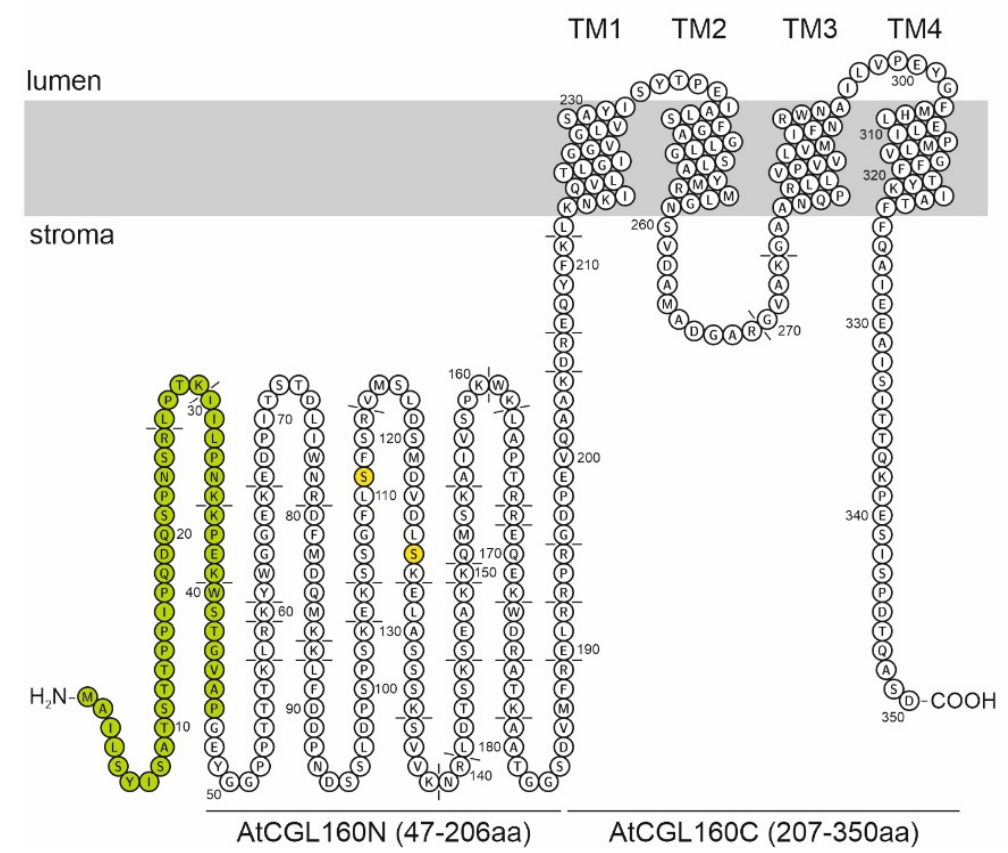

B

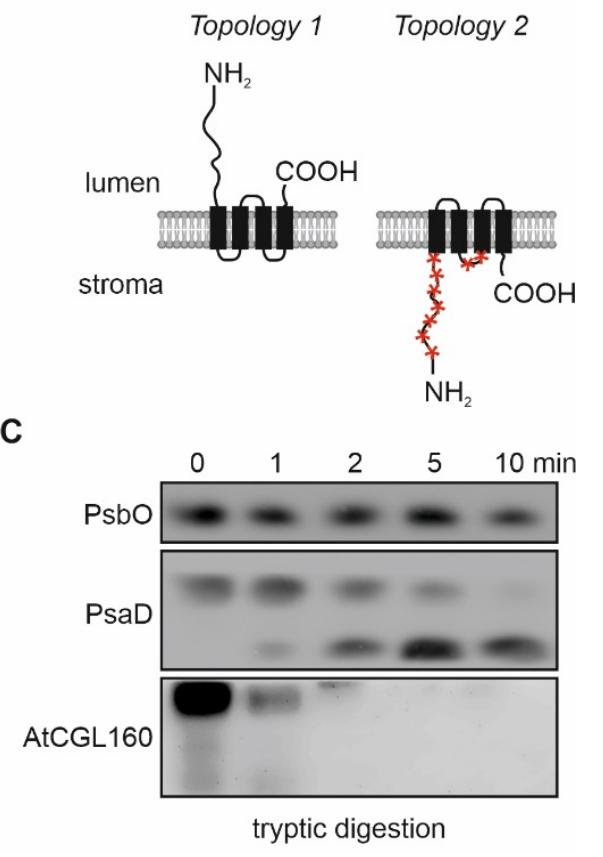

Figure 1. Topology of AtCGL160 and trypsin cleavage-site prediction. A, Transmembrane (TM) domain predictions were obtained from the AtCGL160 UniProt protein accession O82279. Putative trypsin cleavage sites are highlighted in dashed lines and amino-acid positions are indicated. The topology was drawn for the full-length sequence of AtCGL160 including the predicted transit peptide (green) with Protter (Omasits et al., 2014). Two conserved serine residues (S111 and S126) are marked in yellow. B, Representation of two putative AtCGL160 topologies. The four transmembrane domains are indicated as black boxes. Accessible trypsin digestion sites are highlighted by red stars. C, Immunoblot of thylakoid membranes of the WT (Col-0) fractionated by SDS-PAGE, untreated (0 min) or treated with trypsin for 1, 2, 5 and $10 \mathrm{~min}$. Blots were probed with antibodies against the lumenoriented PSII subunit PsbO, the stroma-exposed PSI subunit PsaD and AtCGL160.

Earlier studies have provided experimental evidence for the localization of AtCGL160 to the thylakoid membrane (Rühle et al., 2014; Tomizioli et al., 2014; Fristedt et al., 2015). To gain further insights into the topology of AtCGL160, a protease protection assay was carried out (Fig. 1B, C). In the case of topology 1, all trypsin cleavage sites in AtCGL160 reside in the lumen of the thylakoid and remain fully protected from proteolytic degradation (Fig. 1B). Conversely, the stromal orientation of AtCGL160N predicted for topology 2 would expose trypsin cleavage sites and lead to degradation products of less than $2 \mathrm{kD}$ (Fig. 1A). To test the accessibility of native AtCGL160N, wild-type thylakoids were isolated and treated with trypsin 
for 10 min (Fig. 6B). As expected, the luminal PSII subunit PsbO was not affected by the enzyme, whereas the stromally exposed PSI subunit PsaD was susceptible to the protease. AtCGL160N was also efficiently digested, leaving no detectable proteolytic cleavage products, which is consistent with protrusion of the entire $\mathrm{N}$-terminal domain into the stroma, as shown in topology 2 (Fig. 6A,B).

A

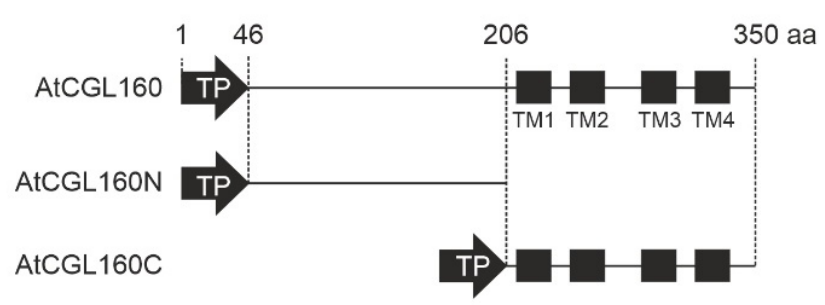

B

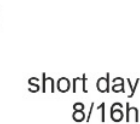
$8 / 16 \mathrm{~h}$
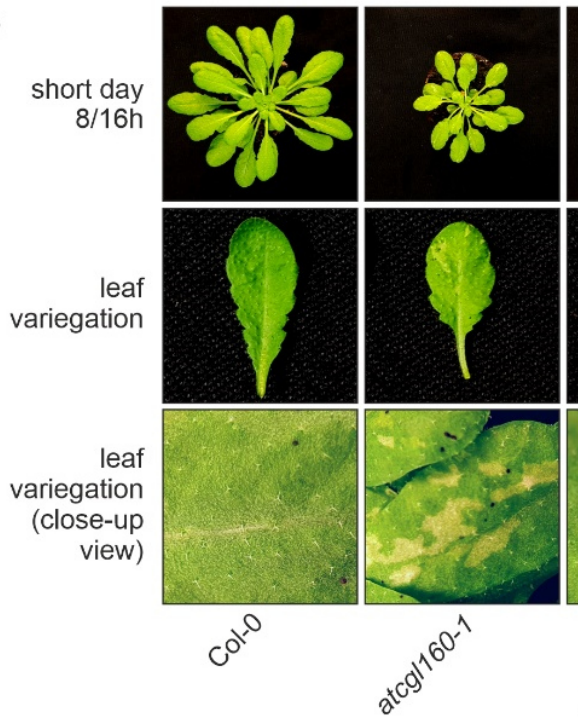

C

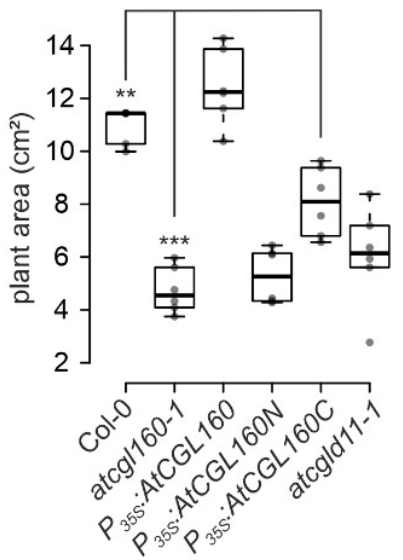

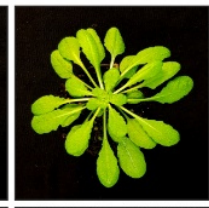
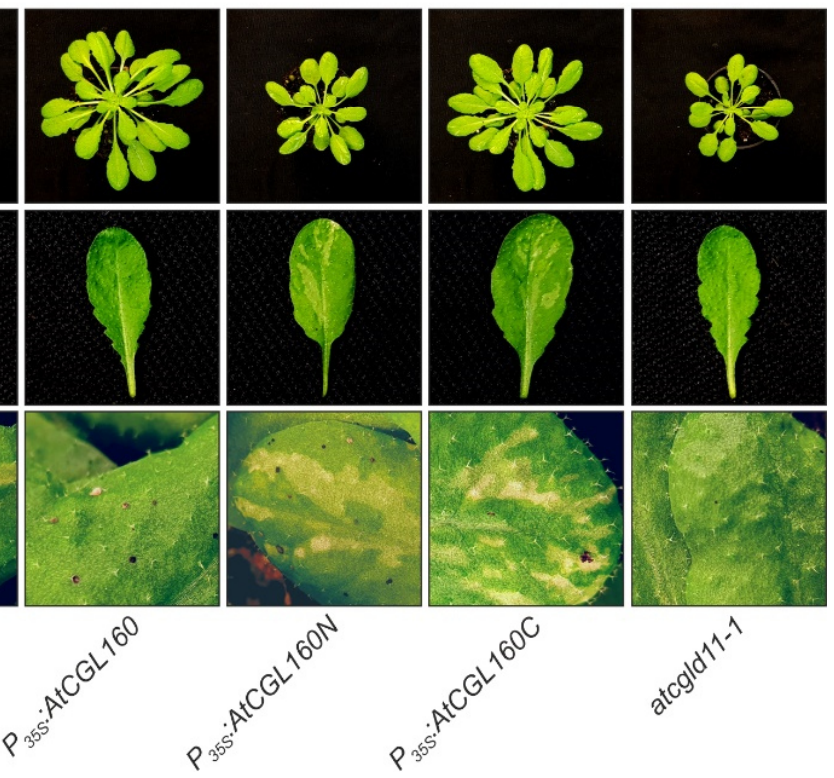
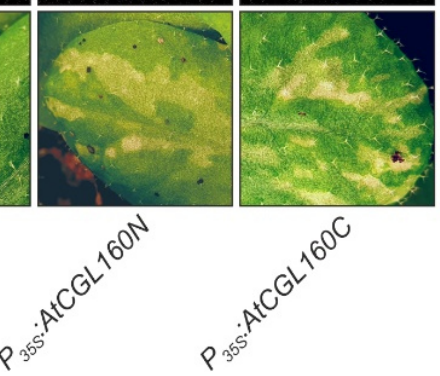

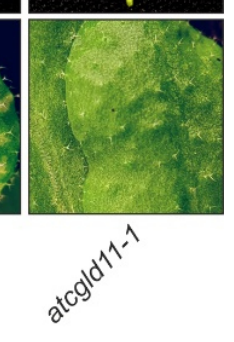

Figure 2. Growth phenotype and leaf variegation of $P_{35 s}$ :AtCGL160, $P_{355}$ :AtCGL160N and $P_{35 s}$ :AtCGL160C plants under short-day conditions. A, Schematic representations of reintroduced AtCGL160 coding sequences. Plants lacking AtCGL160 were transformed with overexpressor constructs harboring the coding sequences for the full-length AtCGL160

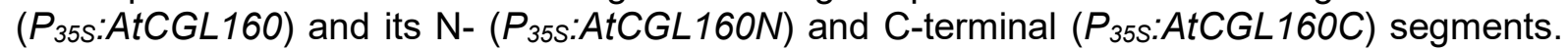
Transcription was under the control of the $35 \mathrm{~S}$ CaMV promoter and targeting to the chloroplast was mediated by the transit peptide of AtCGL160 (TP). Amino-acid positions are indicated and predicted transmembrane domains (TM1-TM4) are schematically shown as black boxes. B,

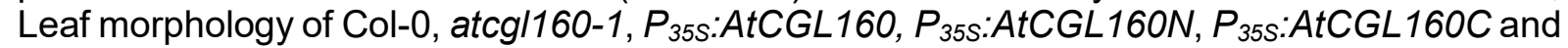
atcgld11-1 plants. C, Leaf areas of 6 individual plants per genotype were determined 4 weeks after germination. The horizontal lines represent the median, and boxes indicate the 25th and 75th percentiles. Whiskers extend the interquartile range by a factor of $1.5 \times$, and outliers are represented by dots. The effect of the deletion of AtCGL160N in $P_{355}$ :AtCGL160C plants on growth under short-day conditions was tested by paired sample t-test (two-sided). Statistically significant differences are marked with asterisks $\left({ }^{*} P<0.05,{ }^{* *} P<0.01\right.$, and $\left.{ }^{* * *} P<0.001\right)$. 
To dissect the function of the N-terminal portion of AtCGL160, three different constructs under control of the $35 \mathrm{~S}$ promoter were cloned, transformed into the atcgl160-1 background and screened for complementation (Fig. 2, Supplemental Fig. 2A). Plants that overexpressed the full-length coding sequence (CDS) of AtCGL160 served as controls ( $P_{35 s}$ :AtCGL160), while the other two genotypes expressed either the CDS of the N-terminal ( $\left.P_{35 s}: A t C G L 160 N\right)$ or the C-terminal segment ( $P_{355}$ :AtCGL160C) of the protein (Supplemental Fig. 2B, C). In the case of $P_{35 s: A t C G L 160 C}$ plants, targeting of the truncated version to chloroplasts was achieved by fusing the CDS of the AtCGL160-derived cTP (1-46 aa) to that of AtCGL160C (Fig. 2A). As was previously demonstrated in complementation analyses with $P_{35 s}$ :AtCGL160-eGFP lines (Rühle et al., 2014), overexpression of the full-length AtCGL160 rescued the atcgl160-1 phenotype (Supplemental Fig. 2A), as indicated by wild-type-like growth and restored leaf morphology under short-day conditions (Fig. 2B, C). P35s:AtCGL160N failed to complement the mutant phenotype (Fig. 2B, C, Supplemental Fig. 2A) and AtCGL160N could not be detected in either stromal or thylakoid extracts (Supplemental Fig. 3). Since AtCGL160N transcripts were present in WT-like amounts in $P_{35 s}$ :AtCGL160N plants (Supplemental Fig. $2 \mathrm{C}$ ), the lack of AtCGL160N is probably due to proteolytic degradation owing to its inability to associate correctly with thylakoids. Nevertheless, $P_{355}$ :AtCGL160N plants were retained and served as an additional AtCGL160 knockout control. $P_{35 s}$ :AtCGL160C plants with similar overexpression rates to $P_{35 s}$ :AtCGL160 plants (Supplemental Fig. 2B, C) were characterized by a significant increase in leaf area compared to the mutant background atcgl160-1, but were growth-retarded with respect to the wild-type control. Interestingly, like atcgl160-1,

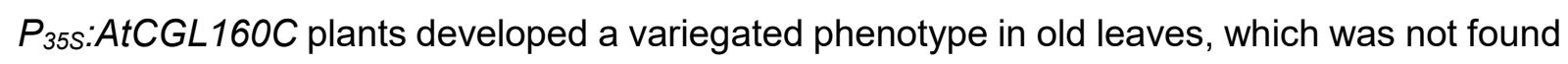
either in the wild type or in the $\mathrm{CF}_{1}$ assembly mutant atcgld11-1 (Grahl et al., 2016) under short-day conditions (Fig. 2B).

To analyze the leaf phenotype in more detail, we carried out electron microscopic analyses of Col-0, atcgl160-1 and $P_{355}$ :AtCGL160C plants (Fig. 3). In these genotypes, the chloroplast ultrastructure in preparations from green leaf sections was unchanged with regard to thylakoid content, curvature and grana organization (Fig. 3 A-F). These observations in atcgl160-1, together with previous ultrastructural analyses of the $\mathrm{CF}_{1}$ assembly mutant line atcgld11-1 (Grahl et al., 2016) and spinach chloroplasts (Daum et al., 2010), support the idea that $\mathrm{CF}_{1-}$ $\mathrm{CF}_{\mathrm{O}}$ complexes are not physically involved in thylakoid curvature formation. Examination of white leaf sections in atcg/160-1 and $P_{35 s}$ :AtCGL160C revealed the absence of thylakoids in plastids, accompanied by the appearance of plastoglobuli in densely packed stromal clusters (Fig. 3 G-J). Furthermore, large vesicles were observed, which also point to increased catabolic activity and degradation processes in atcgl160-1 and $P_{35 s}$ :AtCGL160C plastids. Another finding was the inclusion of mitochondria in degraded plastids, which was also observed, to a lesser extent, in white leaf sectors of $P_{355}$ :AtCGL160C. 


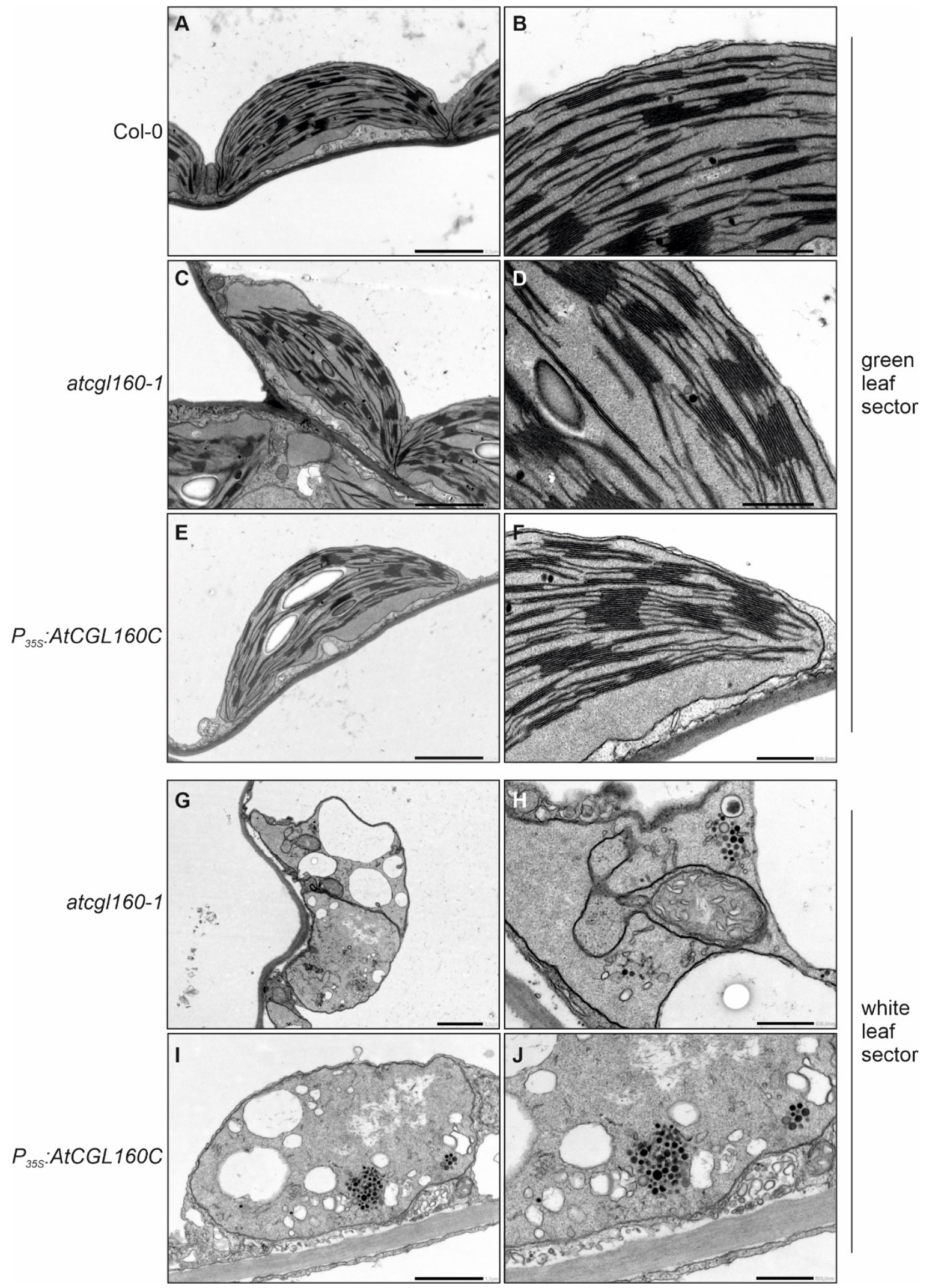

Figure 3. Plastid ultrastructure in white leaf sectors is altered in the absence of AtCGL160N under short-day growth conditions. Electron micrographs of samples from green leaf sections obtained from Col-0 (A, B), atclg160-1 (C, D) and P35s:AtCGL160C (E, F) plants. The ultrastructure of chloroplasts was further examined in samples of white leaf sections obtained from atcg/160-1 (G, H) and $P_{355}$ :AtCGL160C (I, J) plants. The photos on the right show enlargements of the images on the left. The scale bar corresponds to $2 \mu \mathrm{m}$ in $\mathbf{A}, \mathbf{C}$, $\mathbf{E}$, and $\mathbf{G}, 1 \mu \mathrm{m}$ in $\mathbf{I}$ and $0.5 \mu \mathrm{m}$ in $\mathbf{B}, \mathbf{D}, \mathbf{F}, \mathbf{H}$ and $\mathbf{J}$. 
To test whether disruption of AtCGL160N impairs photosynthesis and $\mathrm{CF}_{1}-\mathrm{CF}_{\mathrm{O}}$ activity, measurements of chlorophyll a fluorescence and electrochromic shift (ECS) were carried out on Col-0, atcgl160-1, P35s:AtCGL160, P35s:AtCGL160N, P35s:AtCGL160C and atcgld11-1 plants (Fig. 4A-C). As expected, the $\mathrm{CF}_{1}-\mathrm{CF}_{\mathrm{O}}$ assembly mutants atcg/160-1 and atcgld11-1 showed higher heat dissipation (indicated as non-photochemical quenching, NPQ) and increased proton-motive force $(p m f)$, but lower proton conductivity $\left(\mathrm{gH}^{+}\right)$through the thylakoid membrane compared to the wild-type control. $P_{355}$ :AtCGL160 and $P_{355}$ :AtCGL160N plants displayed similar levels of $\mathrm{NPQ}, p m f$ and $\mathrm{gH}^{+}$to the wild type and the $\mathrm{CF}_{1}-\mathrm{CF}_{\mathrm{O}}$ assembly mutant atcgld11-1, respectively. Notably, photosynthetic parameters were only partially restored in $P_{35 s}$ :AtCGL160C lines.

A

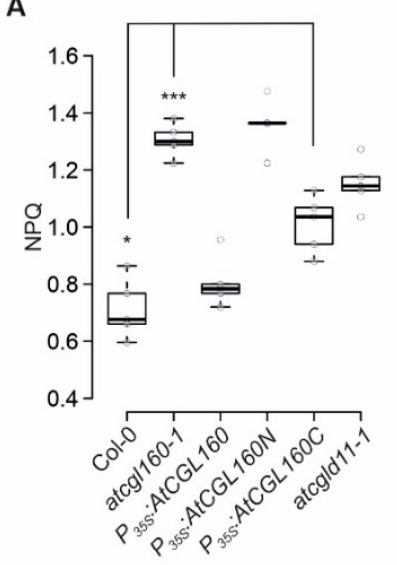

D

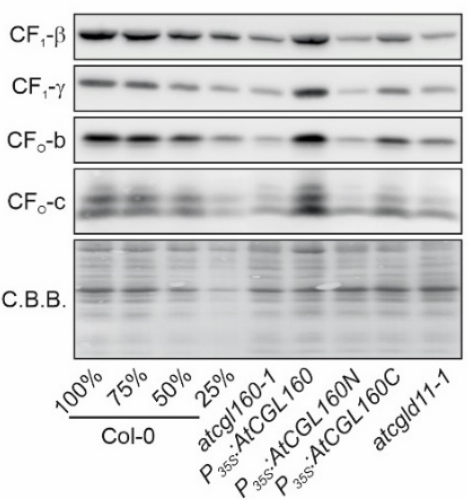

B

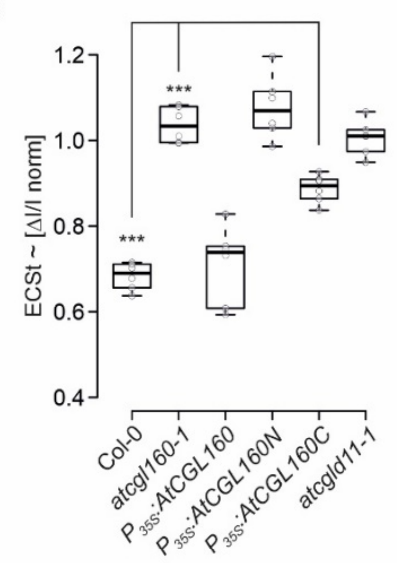

C

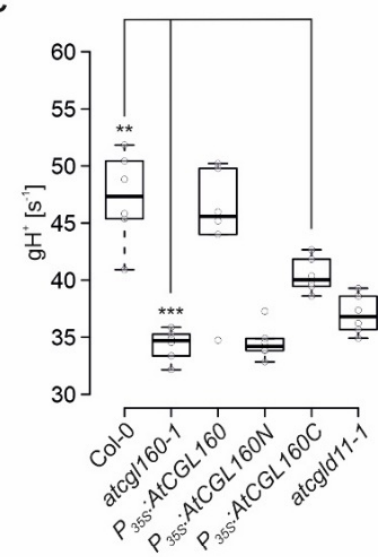

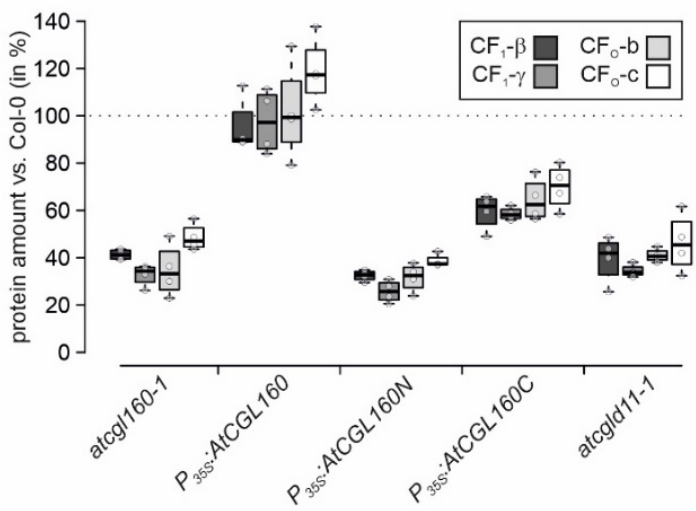

Figure 4. Lack of AtCGL160N perturbs photosynthesis and $C_{1} F_{1}-F_{0}$ integrity. A, Heat dissipation (non-photochemical quenching, NPQ) in Col-0, atcgl160-1, P35s:AtCGL160, $P_{35 s}:$ AtCGL160N, $P_{35 s}$ :AtCGL160C and atcgld11-1 plants grown under short-day conditions. NPQ values from five plants per genotype were determined $105 \mathrm{~s}$ after light induction (145 $\mu \mathrm{mol}$ photons $\mathrm{m}^{-2} \mathrm{~s}^{-1}$ ) using an Imaging-PAM system (Walz). B, Dark-interval relaxation kinetics (DIRK) derived from ECS signals were recorded after 10 min of illumination from six individual plants grown under short-day conditions. Total amplitude of the P515 differential absorption signal was normalized to a single turnover flash 4 min after the ECS measurement. C, Proton conductivity of the thylakoid membrane was determined from ECS signal relaxation rates, which were fitted to a first-order decay function. Calculated rate constants were expressed as $\mathrm{gH}^{+}\left[\mathrm{s}^{-1}\right]$. D, Steady-state levels of immunodetected $\mathrm{CF}_{1}-\mathrm{CF}_{\mathrm{O}}$ marker subunits. After fractionation of thylakoid proteins on SDS-PAGE and transfer to PVDF membranes, blots were probed with antibodies against $\mathrm{CF}_{1-} \beta, \mathrm{CF}_{1-\gamma}, \mathrm{CF}_{\mathrm{O}-\mathrm{b}}$, and $\mathrm{CF}_{\mathrm{O}-\mathrm{C}}$. Coomassie Brilliant Blue 
(C.B.B.) staining is shown as loading control. For quantification, signals from four technical replicates of each marker subunit were normalized to signals detected in Col-0 samples. Horizontal lines represent the median, and boxes indicate the 25th and 75th percentiles. Whiskers extend the interquartile range by $1.5 \times$. The effect of the deletion of AtCGL160N on photosynthetic parameters of $P_{35 s}$ :AtCGL160C plants shown in panels A-C was tested in paired-sample t-tests (two-sided). Statistically significant differences are marked with asterisks $\left({ }^{*} P<0.05,{ }^{* *} P<0.01\right.$, and $\left.{ }^{* * *} P<0.001\right)$.

To assess the integrity of the $\mathrm{CF}_{1}-\mathrm{CF}_{\mathrm{O}}$ complex in thylakoids, marker subunits were immunodetected in atcgl160-1, $P_{35 s}$ :AtCGL160, $P_{35 s: A t C G L 160 N,} P_{35 s: A t C G L 160 C}$ and atcgld11-1 plants, and quantified relative to Col-0 samples (Fig. 4D). Levels of $\mathrm{CF}_{1-} \beta, \mathrm{CF}_{1-\gamma} \gamma$, CFo-b and $\mathrm{CF}_{\mathrm{O}-\mathrm{C}}$ were restored to normal in $P_{355}$ :AtCGL160, but reduced to about $60-65 \%$ of wild-type amounts in $P_{35 s}$ :AtCGL160C plants. Transformation with the $P_{35 s}$ :AtCGL160N construct had no effect on $\mathrm{CF}_{1}-\mathrm{CF}_{\mathrm{O}}$ subunit levels in the atcg/160-1 mutant.

Overall, overexpression of the Atp1/Unc1-like AtCGL160 domain alone (AtCGL160C) in the atcgl160-1 background only partially restored $\mathrm{CF}_{1}-\mathrm{CF}_{\mathrm{O}}$ amounts (Fig. 4D) and activity (Fig. 4C). Consequently, $\Delta \mathrm{pH}$-dependent quenching mechanisms (Fig. 4A) were more highly activated, resulting in downregulation of photosynthesis (Fig. 4B) and growth impairment of $P_{35 s: A t C G L 160 C}$ plants. We deduced from these results that AtCGL160N might also be involved in $\mathrm{CF}_{1}-\mathrm{CF}_{\mathrm{O}}$ assembly at steps other than $\mathrm{CF}_{\mathrm{O}} \mathrm{C}$ ring formation.

\section{Stromal accumulation of $\mathrm{CF}_{1}$ in the absence of AtCGL160N}

To investigate the effects of deletion of AtCGL160N on $\mathrm{CF}_{1}-\mathrm{CF}_{\mathrm{O}}$ assembly, we performed BN/SDS-PAGE (2D-PAGE) analysis on thylakoids isolated from $P_{35 s}$ :AtCGL160 and $P_{35 s: A t C G L 160 C}$ plants grown under short-day conditions. Consistent with the accumulation of $\mathrm{CF}_{1}-\mathrm{CF}_{\mathrm{O}}$ marker subunits in Fig. $4 \mathrm{D}, \mathrm{CF}_{1-}-\beta, \mathrm{CF}_{\mathrm{O}}-\mathrm{b}$ and $\mathrm{CF}_{\mathrm{O}-\mathrm{C}}$ levels were reduced in $P_{35 s}$ :AtCGL160C compared to plants that overexpressed the full-length CDS of AtCGL160 (Fig. 5A). No accumulation of pre-complexes was observed, as amounts of free proteins, and components of the c-ring, $\mathrm{CF}_{1}$ and the holo-complex were reduced uniformly. To assess the assembly status of the c-ring in more detail, we carried out 2D-PAGE with increased amounts of atcgl160-1 and $P_{355}$ :AtCGL160C thylakoids (Fig. 5B). C-ring levels were considerably higher in $P_{35 s}$ :AtCGL160C than in the atcgl160-1 mutant background. We also examined $\mathrm{CF}_{1}$ accumulation in the stroma of Col-0, $P_{35 s}$ :AtCGL160, $P_{355}$ :AtCGL160N, $P_{35 s}$ :AtCGL160C and atcgld11-1 plants (Fig. $5 \mathrm{C}$ ), since $\mathrm{CF}_{1}-\mathrm{CF}_{\mathrm{O}}$ assembly takes place in a modular fashion and involves distinct thylakoid-integral and soluble intermediates. Strikingly, $\mathrm{CF}_{1-} \beta$ and $\mathrm{CF}_{1-\gamma} \gamma$ were enriched about 10-fold in the stroma of atcgl160-1, $P_{35 s}$ :AtCGL160N and $P_{35 s}$ :AtCGL160C, but were detected in close to wild-type levels in $P_{355}$ :AtCGL160 and atcgld11-1 plants. In-depth 2D-PAGE analysis of $\mathrm{CF}_{1}$ intermediates in atcg/160-1, and comparison with results from the co-migration database for photosynthetic organisms (PCom-DB, Takabayashi et al., 2017), 
revealed that in atcgl160-1 stromal $C_{1}-\beta$ and $C_{1}-\gamma$ were predominantly present in an $\alpha_{3} \beta_{3} y \varepsilon$ complex that lacked subunit $\mathrm{CF}_{1}-\delta$ (Supplemental Fig. S4).

We concluded that re-introduction of the transmembrane Atp1/Unc1-like domain of AtCGL160 restores c-ring formation, but leads to an overall reduction in $\mathrm{CF}_{1}-\mathrm{CF}_{\mathrm{O}}$ levels due to a defect in the attachment of $\mathrm{CF}_{1}$ to a membrane-integral $\mathrm{CF}_{\mathrm{O}}$ intermediate.

A

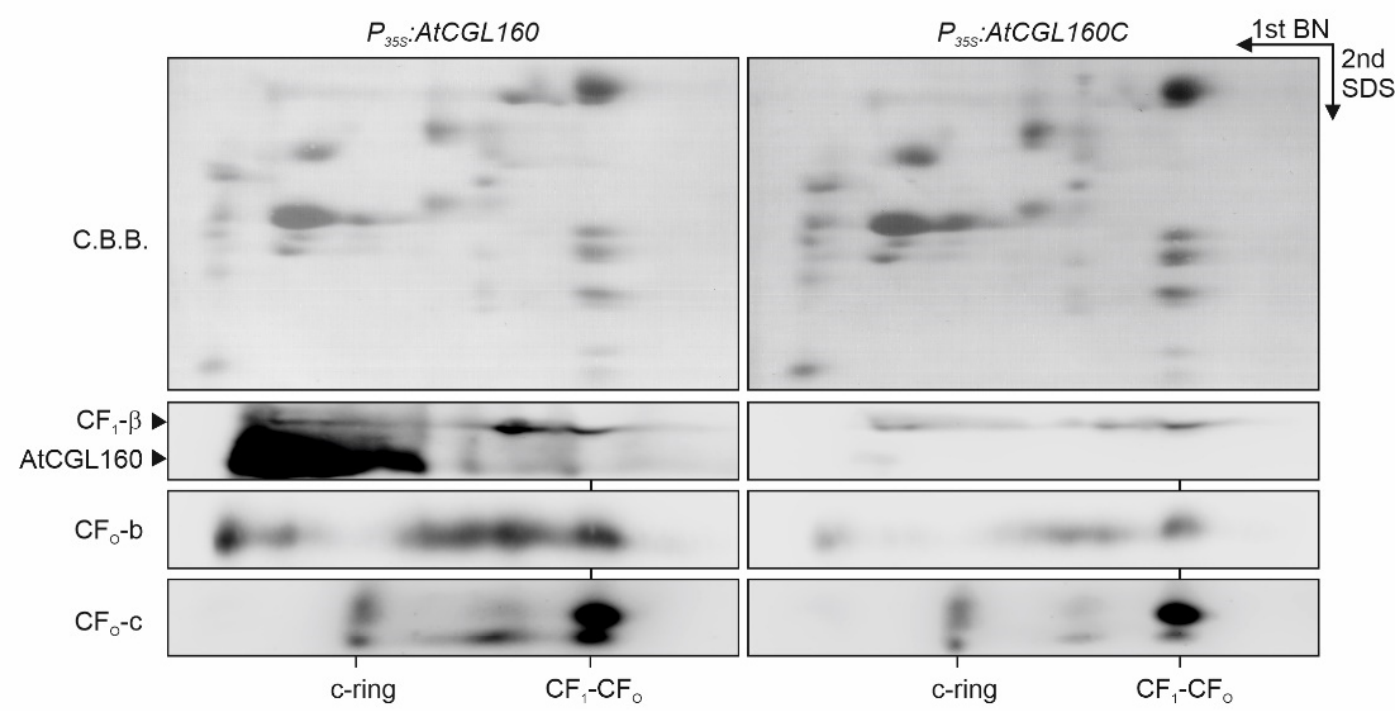

B

C
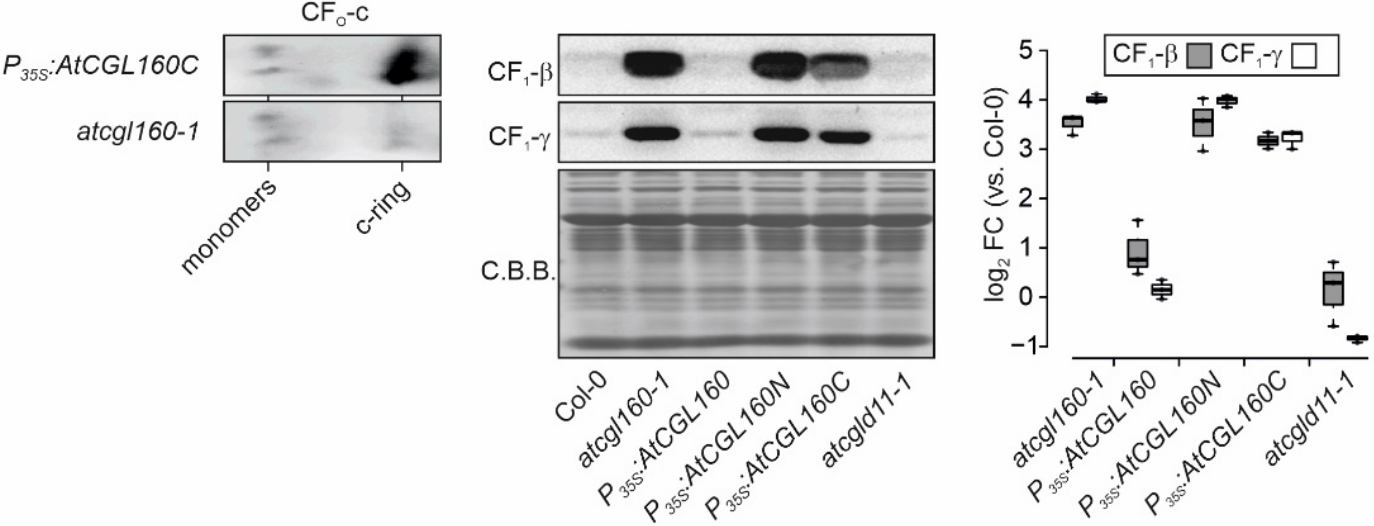

Figure 5. $\mathrm{CF}_{1}-\mathrm{CF}_{\mathrm{O}}$ assembly is perturbed in the absence of AtCGL160N. A, Thylakoid complexes from $P_{35 s}$ :AtCGL160 and $P_{355}$ :AtCGL160C plants were solubilized with $n$-dodecyl $\beta$-D-maltoside ( $1 \%[\mathrm{w} / \mathrm{v}])$ and further separated by Blue-Native (BN, $1^{\text {st }}$ dimension) and SDSPAGE (SDS, $2^{\text {nd }}$ dimension). After protein transfer, PVDF membranes were probed with antibodies against $C_{1}-\beta, C F_{O}-b$ and $C F_{O}-C$, and $C_{1-} \beta$ blots were subsequently exposed to anti-AtCGL160 antibodies. Positions of the ATP synthase holo-complex $\left(\mathrm{CF}_{1}-\mathrm{CF}_{\mathrm{O}}\right)$ and the cring are indicated. Coomassie Brilliant Blue G-250 (C.B.B.) staining of PVDF membranes is shown as loading control. B, C-ring assembly in atcgl160-1 and $P_{355}$ :AtCGL160C plants. Increased amounts of thylakoid complexes (corresponding to $120 \mu \mathrm{g}$ total chlorophyll) were solubilized and fractionated by BN/SDS-PAGE. Blots were probed with an antibody against $\mathrm{CF}_{\mathrm{O}-\mathrm{C}}$. Positions of free $\mathrm{c}$-monomers and the assembled $\mathrm{c}$-ring are indicated. $\mathbf{C}, \mathrm{CF}_{1-} \boldsymbol{\beta}$ and $\mathrm{CF}_{1-Y} \mathrm{Y}$ enrichment in stromal extract, which was isolated from Col-0, atcgl160-1, $P_{35 s}$ :AtCGL160, $P_{35 s}$ :AtCGL160N, $P_{35 s}$ :AtCGL160C and atcgld11-1 rosette leaves. Signals of three $C_{1-} \beta$ and $C_{1-\gamma}$ immunodetection assays were quantified and are shown on a logarithmic scale. Horizontal lines represent the median, boxes indicate the 25th and 75th percentiles and whiskers extend the interquartile range by a factor of $1.5 \times$. 
AtCGL160 interacts physically with $\mathrm{CF}_{1 \text {-containing complexes }}$

A

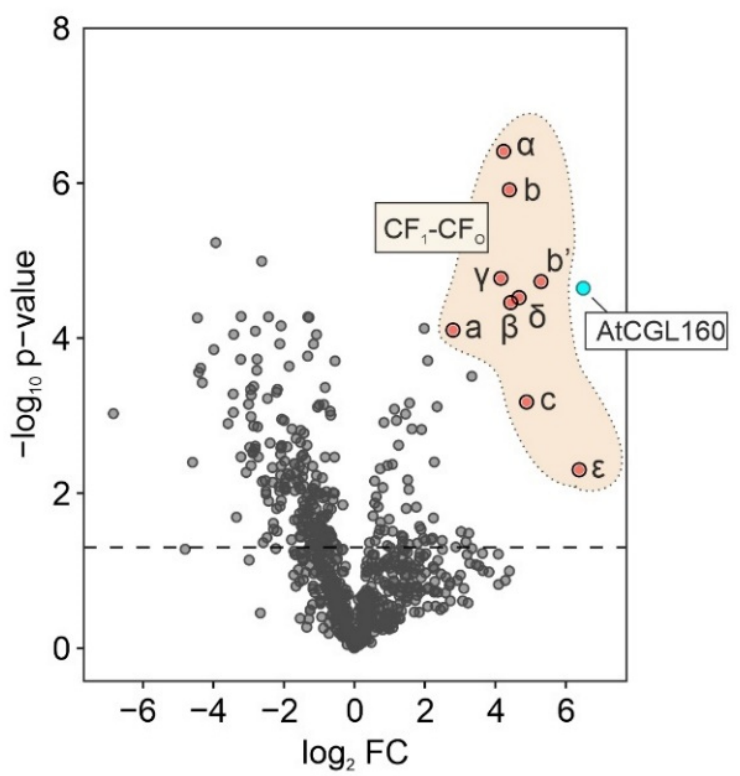

B
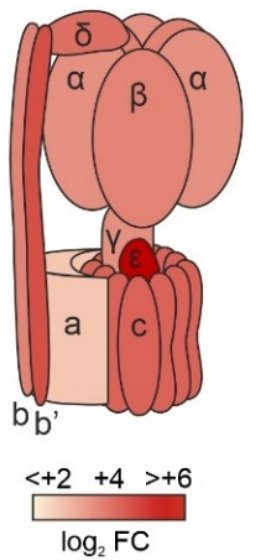

C

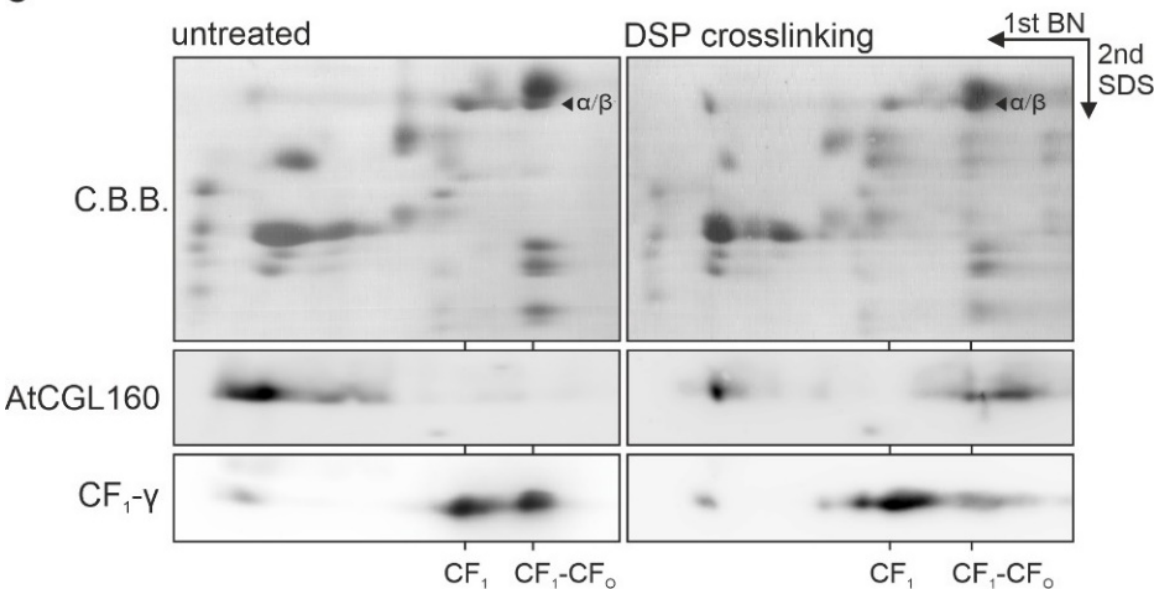

Figure 6. AtCGL160 association with $\mathrm{CF}_{1}$ subunits. $\mathrm{A}$, Co-immunoprecipitation analyses were carried out with solubilized thylakoids isolated from $P_{355}$ :AtCGL160, while $P_{35 s}$ :AtCGL160C plants served as the negative control. Co-immunoprecipitated proteins were further subjected to tryptic digestion, and peptides were analyzed by liquid chromatography coupled to mass spectrometry. Data for differentially enriched proteins are presented in a volcano plot. The relative abundance ( $\log _{2}$ fold change [ $\left.\log _{2} \mathrm{FC}\right]$ ) of proteins coimmunoprecipitated from $P_{35 s}$ :AtCGL160 samples is plotted against their statistically significant enrichment as Benjamini-Hochberg corrected $p$-values $\left(-\log _{10} p\right.$-value). The dashed line indicates a negative $\log _{10} p$-value of 1.5 , and was defined as the threshold for robust reliability of differences in co-immunoprecipitation data. Blue and red dots highlight quantification results for AtCGL160 and $\mathrm{CF}_{1}-\mathrm{CF}_{\mathrm{O}}$ subunits, respectively. B, Schematic representation of differentially enriched subunits in a $\mathrm{CF}_{1}-\mathrm{CF}_{\mathrm{O}}$ cartoon. Relative amounts of co-immunoprecipitated $\mathrm{CF}_{1}-\mathrm{CF}_{\mathrm{O}}$ subunits are shown in colors on a $\log _{2} \mathrm{FC}$ scale from white $\left(\log _{2} \mathrm{FC}<2\right)$ to red $\left(\log _{2} \mathrm{FC}>6\right)$. Co-immunoprecipitation assays were carried out on three independent biological replicates. C, Co-migration of AtCGL160 with $\mathrm{CF}_{1}-\mathrm{CF}_{\mathrm{O}}$ in crosslinking experiments. Two-dimensional BN/SDS-PAGE analysis was used to compare untreated thylakoid extracts of the WT (Col-0) with extracts that had been crosslinked with dithiobis(succinimidyl propionate) (DSP). Blots of the second dimension were probed with antibodies against AtCGL160 and $\mathrm{CF}_{\mathrm{O}} \mathrm{Y}$. The positions of $\mathrm{CF}_{1}-\mathrm{CF}_{\mathrm{O}}$, the $\mathrm{CF}_{1}$ intermediate, and the free protein fraction are indicated based on the mobility of $\alpha / \beta$ on the C.B.B. stained gel. 
To pinpoint the role of AtCGL160 in the recruitment of $\mathrm{CF}_{1}$ to a membrane-integral $\mathrm{CF}_{\mathrm{O}}$ intermediate, protein interactions were assessed in co-immunoprecipitation (co-IP) assays (Fig. 6B). Quantitative data for precipitated proteins were obtained by tryptic digestion and subsequent peptide-fragment analysis using liquid chromatography coupled to mass spectrometry. Since the commercially available AtCGL160 antibody (Agrisera AS12 1853) displayed non-specific binding to either $\mathrm{CF}_{1-\alpha} \alpha$ or $\mathrm{CF}_{1-}-\beta$ (Supplemental Fig. 3), an AtCGL160 antibody with no significant cross-reactions to other thylakoid proteins was generated (Supplemental Fig. 3). In the first step of antibody production, the N-terminal part of AtCGL160 (AtCGL160 29 -206aa) was fused to the maltose-binding protein and injected into rabbits. In the second step, antibodies specific for AtCGL160 $29-206$ aa were affinity-purified from rabbit antisera using an immobilized fusion protein consisting of AtCGL160 ${ }_{29-206 a a}$ and glutathione Stransferase. As expected, when the resulting antibody fraction was tested in immunodetection assays, it showed only one distinct signal in the WT sample, which was enriched in extracts of $P_{355}$ :AtCGL160, but was absent in both the atcg/160-1 mutant and in $P_{35 s}$ :AtCGL160C samples (Supplemental Fig. 3).

Next, NP40-solubilized thylakoid proteins from $P_{35 s}$ :AtCGL160 plants grown under short-day conditions were chosen as co-IP input and pulled-down protein amounts were compared to those recovered in co-IP experiments carried out on thylakoid protein extracts of $P_{35 s}$ :AtCGL160C. Plants devoid of AtCGL160 were not considered for use as negative controls, since the reduction in $\mathrm{CF}_{1}-\mathrm{CF}_{\mathrm{O}}$ levels observed in atcgl160-1 (and $P_{35 \mathrm{~s}}$ :AtCGL160N) (Fig. 4D) might lead to misinterpretation of differential co-IP experiments. As expected, AtCGL160 was pulled down efficiently from $P_{35 s}$ :AtCGL160C extracts ( $\log _{2}$ FC 6.5). Moreover, all $\mathrm{CF}_{1}-\mathrm{CF}_{0}$ subunits were identified in co-IPs (Fig. 6A,B) with high differential enrichment levels for the subunits $\alpha, \beta, \gamma, \delta, \varepsilon, b$, b' and $c\left(\log _{2} F C>4.4\right)$. Subunit $\mathrm{CF}_{\mathrm{O}}$-a was co-immunoprecipitated at lower levels ( $\log _{2} \mathrm{FC} \sim 2.8$ ). The pull-down of $\mathrm{CF}_{1}$ subunits was confirmed by immunodetection assays of the two marker subunits $C_{1}-\beta$ and $C F_{1-\gamma}$, which were only detectable in co-IP output fractions obtained from $P_{35 s}$ :AtCGL160 samples (Supplemental Fig. 5). Other known $\mathrm{CF}_{1}-\mathrm{CF}_{\mathrm{O}}$ assembly factors were not coimmunoprecipitated (Supplemental Table 3), indicating that AtCGL160 is associated with a late $\mathrm{CF}_{1}-\mathrm{CF}_{\mathrm{O}}$ assembly stage or the fully assembled complex from which other auxiliary factors had already dissociated. To confirm the association of AtCGL160 with CF $_{1}$-containing complexes, crosslinking experiments were also carried out (Fig. 6C). To this end, thylakoid membranes of wild-type plants were treated with the crosslinker dithiobis(succinimidyl propionate) (DSP), and subsequently subjected to 2D-PAGE and immunodetection of AtCGL160 and CF 1 -CFo marker subunits. In analyses with untreated thylakoid samples, AtCGL160 migrated predominantly in 
the monomer fraction. After crosslinking, AtCGL160 could be detected at a molecular mass range which corresponded to that of the $\mathrm{CF}_{1}-\mathrm{CF}_{\mathrm{O}}$ holo-complex.

In summary, co-IP of all $\mathrm{CF}_{1}-\mathrm{CF}_{\mathrm{O}}$ subunits with an AtCGL160-specific antibody, together with the observation that AtCGL160 co-migrated with the $\mathrm{CF}_{1}-\mathrm{CF}_{\mathrm{O}}$ holo-complex after DSP crosslinking, corroborates the involvement of AtCGL160 in the functional integration of $\mathrm{CF}_{1}$ into the holo-complex at a late step in $\mathrm{CF}_{1}-\mathrm{CF}_{0}$ assembly.

AtCGL160N interacts with $\mathrm{CF}_{1-\beta} \beta$ in yeast two-hybrid assays

A

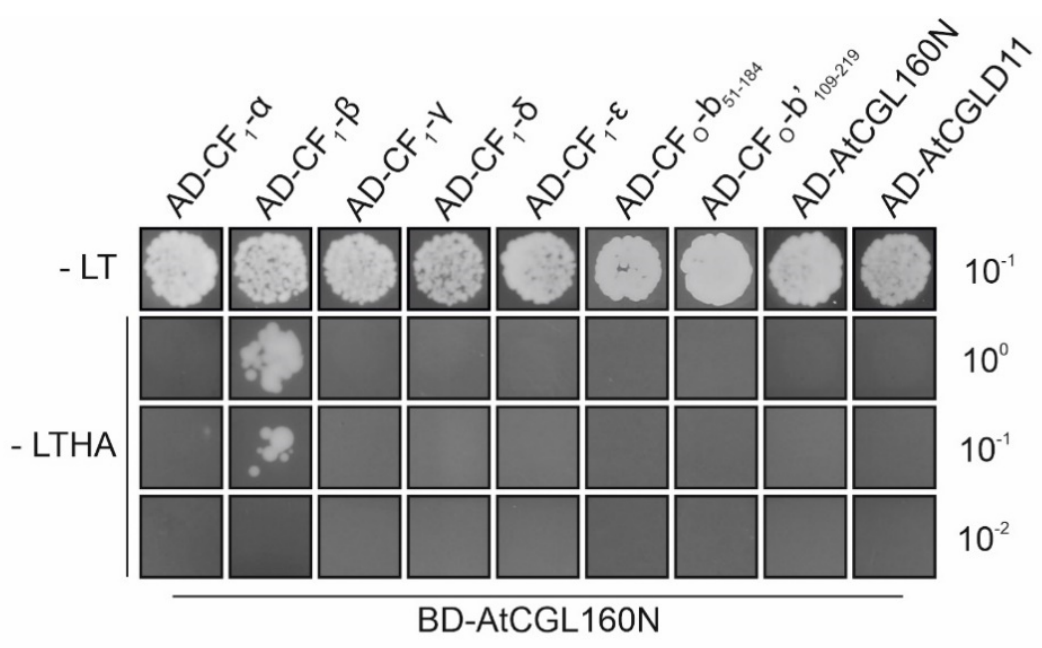

B
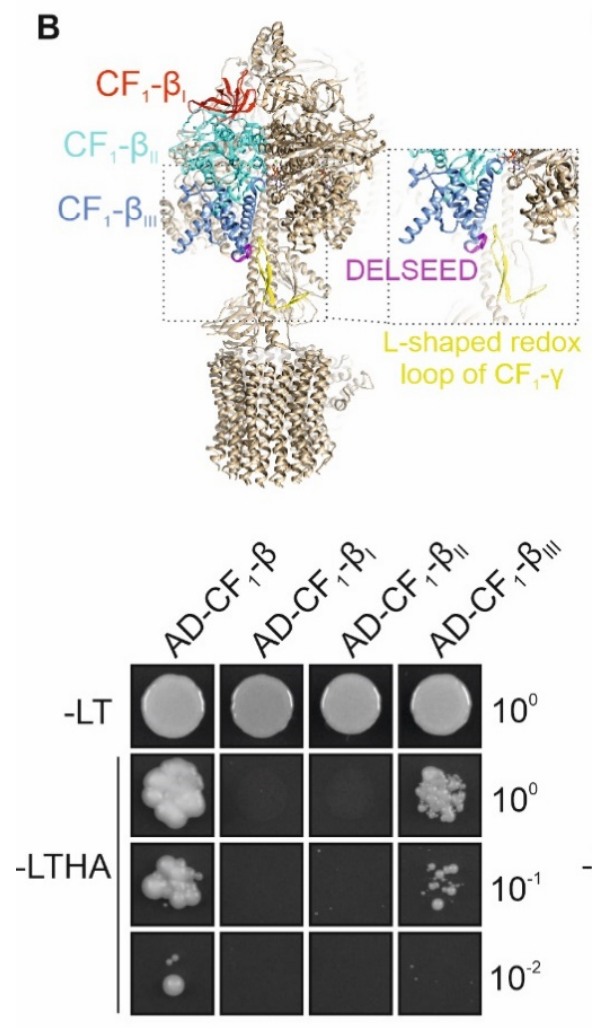

BD-AtCGL160N

C
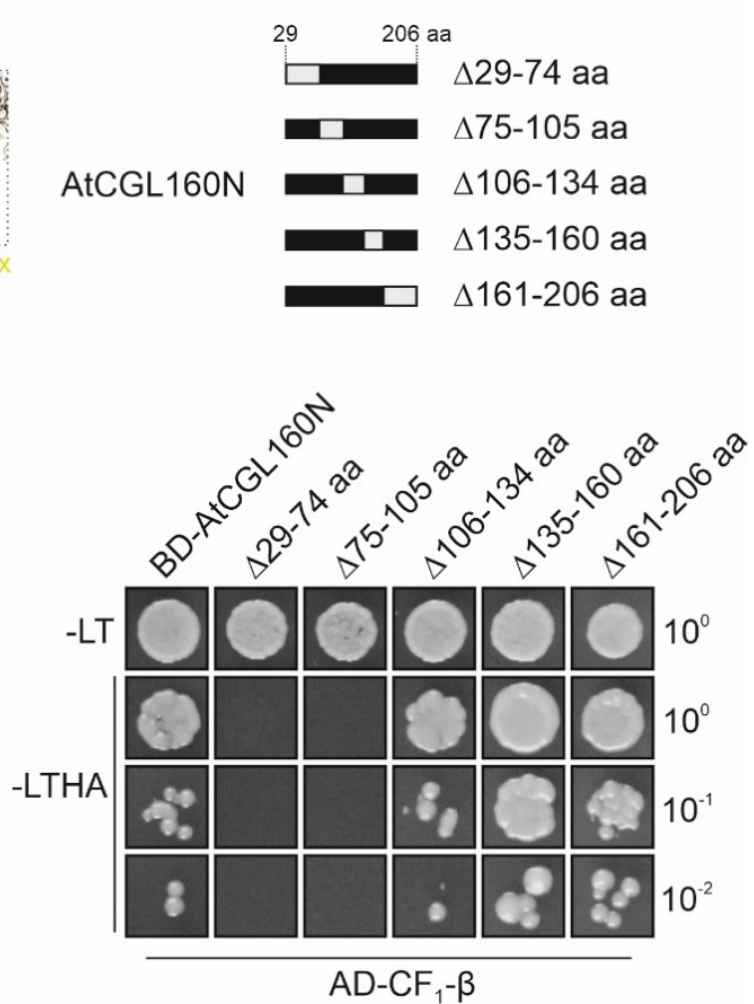

Figure 7. AtCGL160N interaction studies in yeast two-hybrid assays. A, Interactions of AtCGL160 with $\mathrm{CF}_{1}-\mathrm{CF}_{\mathrm{O}}$ structural components exposed on the stromal side of thylakoids were tested by transformation of a construct that fuses AtCGL160N to the GAL4 DNA-binding 
domain (BD-AtCGL160). Cells were then co-transformed with constructs coding for GAL4

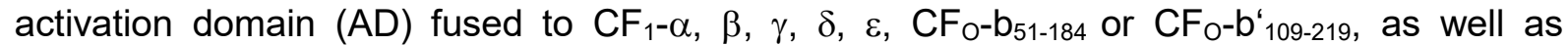
AtCGL160N or AtCGLD11. B, Interaction of AtCGL160N with structural domains of $C_{1-}-\beta$. Yeast cells carrying a construct coding for BD-AtCGL160 were transformed with constructs coding for $A D-C F_{1}-\beta_{l}, A D-C F_{1}-\beta_{\| I}$, and $A D-C F_{1}-\beta_{I I I}$. Structural domains of the $C_{1}-\beta$ are colored in red (domain I), turquoise (domain II), and blue (domain III). The conserved DELSEED motif is shown in purple, and the L-shaped redox loop of $\mathrm{CF}_{1-\gamma}$ in yellow. The atomic model of $\mathrm{CF}_{1-}$ $\mathrm{CF}_{\mathrm{O}}$ was obtained from the PDB database (ID: 6fkh, Hahn et al. (2018)) and formatted with ChimeraX (Pettersen et al., 2021). C, Mapping of the AtCGL160N interaction site. Consecutive regions (grey boxes) coding for segments of the soluble AtCGL160 domain were omitted from the BD-AtCGL160N vector and co-transformed with $A D-C_{1}-\beta$ into competent yeast cells. Transformations were verified by plating on permissive medium lacking Leu and Trp (-LT). Interactions were then tested on selective medium (-Leu/-Trp/-His/-Ade, [-LTHA]) by plating equal numbers of yeast cells in serial dilutions $\left(10^{0}, 10^{-1}\right.$, and $\left.10^{-2}\right)$.

Interactions between the stroma-oriented AtCGL160N domain and individual $\mathrm{CF}_{1}$ subunits were further examined by yeast two-hybrid experiments (Fig. 7). A construct coding for a fusion of AtCGL160N 29 -206aa to the GAL4-binding domain (BD) was co-transformed into yeast cells together with constructs coding for GAL4 activation domain (AD) fusions to all $\mathrm{CF}_{1}$ subunits $(\alpha, \beta, \gamma, \delta, \varepsilon)$. Moreover, BD-AtCGL160N interaction was tested with AD fusions to the soluble parts of the stator subunits $b$ and b', AtCGL160N, and CF $_{1}$ assembly factor AtCGLD11. As a result, only yeast cells carrying constructs for $A D-C_{1}-\beta$ and BD-AtCGL160N could grow on selective medium (Fig. 7A). To narrow down the $\mathrm{CF}_{1-} \beta$ interaction site, additional $A D$ fusion constructs were cloned that encoded three different $C_{1-} \beta$ subdomains (Fig. 7B) defined in earlier studies (Groth and Pohl, 2001; Zhang et al., 2016). Domain I comprises a thylakoid-distal $\beta$-barrel structure and interacts with $\mathrm{CF}_{1-}-\delta$. Domain II harbors the catalytic site involved in ATP generation or hydrolysis. The thylakoid-proximal domain III contains the conserved "DELSEED" motif, which is required for $\mathrm{CF}_{1}-\mathrm{\gamma} / \varepsilon$-dependent regulation of ATP hydrolysis and synthase activity (Kanazawa et al., 2017; Hahn et al., 2018). When tested on restrictive medium, only cells harboring $A D-C_{1}-\beta_{\text {III }}$ together with $B D-A t C G L 160 N$ could grow. In a reciprocal approach, coding sequences of AtCGL160N were deleted successively from the BD-AtCGL160N construct $(\Delta 29-74, \Delta 75-105, \Delta 106-134, \Delta 135-160$ and $\Delta 161-206$ aa) and tested for $A D-C_{1}-\beta$ interaction in yeast cells (Fig. $7 C$ ). Only the $\Delta 29-74$ and $\Delta 75-105$ deletions resulted in an absence of growth, while yeast strains with deletion constructs of $\Delta 106-134$, $\Delta 135-160$ and $\Delta 161-206$ aa were able to proliferate on selective medium (Fig. $6 \mathrm{~B}$ ). Thus, the interaction between AtCGL160 and CF $_{1}$ involves AtCGL160 $29-105$ and the thylakoid-proximal domain of $\mathrm{CF}_{1}-\beta_{\mathrm{III}}$, while the phosphorylation hotspot identified in the protein segment 106-134 aa (Fig. $1 \mathrm{~A})$ is dispensable for the interaction. 


\section{Discussion}

\section{AtCGL160N recruits a stromal $\alpha_{3} \beta_{3} y \varepsilon$ complex for late $C_{1}-C F_{0}$ assembly steps}

Despite structural similarities and comparable subunit compositions, the number of known assembly factors for ATP synthases is markedly higher in chloroplast than in bacterial systems (reviewed in Zhang et al., 2020). Moreover, in plants the Atp1/Uncl-related CGL160 assembly factor has acquired an $\mathrm{N}$-terminal domain that is specific for the green lineage. Thus, the expanded molecular inventory for $\mathrm{CF}_{1}-\mathrm{CF}_{\mathrm{O}}$ assembly in chloroplasts might reflect the need for tight post-translational control of $\mathrm{CF}_{1}-\mathrm{CF}_{\mathrm{O}}$ formation, since the complex plays a central role in pmf utilization and regulation of photosynthesis (reviewed in Avenson et al., 2005). In this context, an important finding of previous studies was that disruption of full-length AtCGL160 (Rühle et al., 2014; Fristedt et al., 2015) was more detrimental to levels of functional ATP synthase than the loss of Atp1/Uncl in bacteria (Gay, 1984; Liu et al., 2013). Furthermore, we show here that expression of $P_{355}$ :AtCGL160C in plants that lack AtCGL160N only partially restores $\mathrm{CF}_{1}-\mathrm{CF}_{\mathrm{O}}$ levels and activity (Fig. 4). These observations prompted us to investigate the molecular function of the green-lineage-specific AtCGL160N in the $\mathrm{CF}_{1}-\mathrm{CF}_{\mathrm{O}}$ assembly process in more detail.

Several lines of evidence suggest that the N-terminal domain of AtCGL160 recruits a stromal $\mathrm{CF}_{1}$ intermediate, while the $\mathrm{C}$-terminal segment participates in $\mathrm{C}_{14}$-ring assembly: (i) AtCGL160N protrudes into the stroma, as deduced from protease protection assays (Fig. 1); (ii) formation of the $\mathrm{C}_{14}$ ring is restored in the presence of AtCGL160C alone, but $\mathrm{CF}_{1}$ accumulates strongly in the stroma in the absence of AtCGL160N (Fig. 5), (iii) $\mathrm{CF}_{1}$ subunits are differentially enriched in co-IP analyses performed with solubilized thylakoids isolated from $P_{35 s}$ :AtCGL160 plants (Fig. $6 \mathrm{~A}, \mathrm{~B}$ ), (iv) AtCGL160 co-migrates with a large complex after DSPmediated crosslinking (Fig. $6 \mathrm{C}$ ) and (v) AtCGL160N interacts with $\mathrm{CF}_{1-\beta} \beta$ in yeast two-hybrid experiments (Fig. 7).

A role for AtCGL160 in the incorporation of $\mathrm{CF}_{1}$ into the holocomplex was previously proposed by Fristedt et al. (2015). This assumption was based on the observations that AtCGL160 comigrated with $\mathrm{CF}_{1}$ subcomplexes in $\mathrm{BN} / \mathrm{SDS}-\mathrm{PAGE}$ analyses and could be cross-linked to $\mathrm{CF}_{1}$ subunits in wild-type protein samples. However, we detected AtCGL160 predominantly in the monomer fraction in untreated thylakoid preparations in this study (Fig. 6C), as well as in previous work (Rühle et al., 2014) - and co-migration of AtCGL160 with high-molecular-mass complexes was only observed after thylakoid proteins had been crosslinked with DSP (Fig. 6C). Furthermore, the commercially available AtCGL160 antibody (AS12 1853, Agrisera) employed in the study of Fristedt et al. (2015) was found here to cross-react strongly with $\mathrm{CF}_{1 \text { - }}$ $\alpha$ or $\mathrm{CF}_{1-} \beta$ (Supplemental Fig. 3), which complicates the interpretation of one-dimensional comigration and crosslinking experiments in the absence of appropriate controls. Therefore, $a$ 
new antibody was generated that does not cross-react with $\mathrm{CF}_{1}-\mathrm{CF}_{O}$ subunits and thus provides a reliable means of probing the molecular interactions of AtCGL160 (Supplemental Fig. S3).

Besides CGL160, ALB4 - a member of the bacterial ALB3/Oxa1/YidC protein insertase family - was previously proposed to participate in the linkage of a $\mathrm{CF}_{1}$ to a $\mathrm{CF}_{\mathrm{O}}$ assembly module (Benz et al., 2009). Another study provided evidence that ALB4 and its paralog ALB3 physically interact with each other, and show significant functional overlap in the membrane insertion of subunits of the Cyt $b_{6} f$ complex (Trosch et al., 2015). Moreover, alleles of ALB4 (STIC1) have been identified as suppressors of the chloroplast protein import mutant tic40 (Bedard et al., 2017), and ALB4/STIC1 and STIC2 were shown to act together in thylakoid protein targeting in a pathway that also involves cpSRP54 and cpFtsY. In our study, we did not identify ALB4/STIC1 in co-IP experiments with anti-AtCGL160 antibodies (Fig. 6, Supplemental Table S1) and amounts of thylakoid-associated $C_{1-} \beta$ in atalb4-1 mutants (SALK_136199C) grown under short-day conditions were unaltered (Supplemental Fig. S6). Thus, ALB4/STIC1 does not act in concert with CGL160 in late stages of $\mathrm{CF}_{1}-\mathrm{CF}_{\mathrm{O}}$ assembly, but serves as a general thylakoid protein biogenesis factor involved in folding or assembly of a specific subset of transmembrane proteins (Bedard et al., 2017).

\section{AtCGL160 is critical for chloroplast development in the dark}

It has long been thought that the hydrolytic activity of $\mathrm{CF}_{1}-\mathrm{CF}_{\mathrm{O}}$ needs to be inactivated in the dark to prevent futile ATP depletion (Ort and Oxborough, 1992). However, analysis of the constitutively redox-activated $y$-subunit mutant gamera, in which a 'dark $p m f$ is maintained, revealed increased stability of photosynthetic complexes upon prolonged darkness, suggesting that a certain degree of ATPase activity may be beneficial during the night (Kohzuma et al., 2017). Concomitantly, several processes have been proposed to depend on the maintenance of a dark pmf. These include thylakoid protein transport via the Tat- and Secdependent pathways, modulation of protease activity and ion homeostasis in the chloroplast. In this regard, a remarkable influence of AtCGL160 disruption on leaf variegation (Fig. 2) and chloroplast development (Fig. 3) was observed exclusively under short-day conditions. Surprisingly, this phenotype was not detectable in atcgld11-1 plants with a defect in $\mathrm{CF}_{1}$ assembly and reduced amounts of $\mathrm{CF}_{1}-\mathrm{CF}_{\mathrm{O}}$ comparable to those in atcgl160-1 (Fig. 4D). However, the leaf phenotype correlated with the accumulation of a $\mathrm{CF}_{1}$ intermediate in the stroma (Fig. 5C). Thus, AtCGL160-mediated $\mathrm{CF}_{1}$ recruitment might also be critical in preserving the dark pmf at night. Alternatively, stroma-enriched $\mathrm{CF}_{1}$ complexes (Fig. 5C) could alter the chloroplast ATPIADP ratio by excessive hydrolytic activity, and disturb ATPdependent nocturnal processes that ultimately lead to premature chloroplast degradation (Fig. $3)$. 


\section{AtCGL160 is a central $\mathrm{CF}_{1}-\mathrm{CF}_{0}$ assembly factor with multiple functions}

Assembly of membrane-embedded ATP synthase modules and their subsequent association with $F_{1}$ subcomplexes are critical steps in bacterial and organellar ATP synthase biogenesis, as premature formation of the proton-translocating channel between the c-ring and the asubunit (equivalent to the ATP9 ring and the ATP6 subunit in mitochondria) can lead to uncontrolled dissipation of the pmf (Birkenhäger et al., 1999; Franklin et al., 2004), and only efficient integration of $F_{1}$ triggers ATP production. In this context, molecular aspects of the assembly processes were recently elucidated for bacterial (reviewed in Deckers-Hebestreit, 2013), as well as yeast and human mitochondrial ATP synthases (reviewed in Song et al., 2018). One significant outcome was that, while ATP synthase assembly pathways and the repertoire of auxiliary factors differ among these systems, formation of the proton-translocating unit during the final assembly steps is common to all of them. in c-ring formation and the recruitment of a $\mathrm{CF}_{1}$ intermediate. In fact, these two events were suggested to proceed sequentially in the assembly of bacterial ATP synthases (DeckersHebestreit, 2013). Since an $E$. coli strain lacking subunit $\delta$ accumulates a $c_{10} \alpha_{3} \beta_{3} \gamma \varepsilon$ subcomplex, it is assumed that cytoplasmic $F_{1}$ first binds to the $c_{10}$ ring, and $c_{10} \alpha_{3} \beta_{3} \gamma \varepsilon$ associates with the $a_{2}$ module in a $\delta$-dependent manner in the final assembly step (Hilbers et al., 2013). By analogy with the bacterial assembly pathway, AtCGL160 may facilitate the integration of a stator assembly module into the holo-complex. Indeed, the interaction of AtCGL160C with CFo-b has been demonstrated in split-ubiquitin assays (Rühle et al., 2014). Moreover, $\mathrm{CF}_{\mathrm{O}}$-a was less highly enriched in co-IP analyses than other $\mathrm{CF}_{1}-\mathrm{CF}_{\mathrm{O}}$ subunits (Fig. $6 \mathrm{~A}, \mathrm{~B}$ ), which might argue for the release of AtCGL160 after functional incorporation of $\mathrm{CF}_{\mathrm{O}} \mathrm{a}$ in the final steps of $\mathrm{CF}_{1}-\mathrm{CF}_{\mathrm{O}}$ assembly. In this scenario, AtCGL160 could act as a placeholder to prevent the premature formation of proton-translocating intermediates. A similar function has been described for the INA complex in yeast mitochondria, which binds to the c-ring, but also to a distinct assembly intermediate consisting of ATP6, ATP8, ATP10, ATP23, peripheral stalk subunits and the $F_{1}$ domain (Naumenko et al., 2017). This ensures that the c-ring and subunit ATP6 are assembled into the proton-conducting unit in a controlled manner. However, due to a generally low turnover rate of $\mathrm{CF}_{1}-\mathrm{CF}_{\mathrm{O}}$ assembly (reviewed in Schöttler et al., 2014) and inefficient detection of distinct thylakoid-integral intermediates, a robust $\mathrm{CF}_{\mathrm{O}}$ assembly map is still lacking, and 'true' stator-containing assembly modules have not been described so far.

508 Nevertheless, a straightforward assembly mechanism for the recruitment of $\mathrm{CF}_{1}$ can be derived 509 from our study. After AtCGL160-assisted ring formation (Rühle et al., 2014), the stromally oriented AtCGL160N (Fig. 1) binds to a $C F_{1}$ intermediate consisting of $\alpha_{3} \beta_{3} \gamma \varepsilon$ but not subunit 
$\delta$ (Fig. 5C, Supplemental Fig. S4). Recruitment is mediated through interaction of AtCGL160 $29-$ 105 with subunit $\mathrm{CF}_{1-}-\beta$; thus, the phosphorylatable AtCGL160 segment is dispensable for the interaction (Fig. 7). Since AtCGL160 can be cross-linked to high-molecular-mass complexes that are larger than $C_{1}$ (Fig. 6C), AtCGL160 might remain attached to a putative $C_{14} \alpha_{3} \beta_{3} \gamma \varepsilon$ or ${ }^{b b}{ }^{\prime}{ }_{14} \alpha_{3} \beta_{3} \gamma \varepsilon$ intermediate. Its release could then be triggered by the incorporation of subunit $\mathrm{CF}_{\mathrm{O}-\mathrm{a}}$ or $\mathrm{CF}_{1-} \delta$ in the final assembly steps. At this stage, we cannot rule out the possibility that AtCGL160N might have regulatory functions beyond $\mathrm{CF}_{1}$ recruitment, as it interacts with the thylakoid-proximal domain III of $\mathrm{CF}_{1}$ -

$519 \beta$, which contains the conserved DELSEED motif (Fig. 7B). Several regulatory mechanisms 520 have been elucidated in which the subunit $\beta$ and the DELSEED motif are implicated. For 521 instance, the autoinhibitory subunit $\varepsilon$ interacts with the DELSEED motif in bacteria (Tanigawara 522 et al., 2012; Sobti et al., 2016), whereas in bovine (Cabezon et al., 2003) and yeast 523 mitochondria (Robinson et al., 2013), the small protein $\mathrm{IF}_{1}$ inhibits ATPase activity by binding 524 at the $\alpha / \beta$ interface. In plants, a regulatory mechanism controls $\mathrm{CF}_{1}-\mathrm{CF}_{0}$ activity involving also 525 the DELSEED and an L-shaped, two $\beta$-hairpin containing motif with two conserved redox526 sensitive cysteines in the $\mathrm{CF}_{1-Y} \mathrm{Y}$ subunit (Hahn et al., 2018). By analogy with the role of $\mathrm{IF}_{1}$, 527 which was shown to inhibit ATPase activity during the assembly of human mitochondrial ATP 528 synthases (He et al., 2018), AtCGL160N may regulate ATPase activity during $\mathrm{CF}_{1}-\mathrm{CF}_{\mathrm{O}}$ assembly via an as yet unknown mechanism. 


\section{Methods}

\section{Bioinformatics Sources}

Protein and gene sequences were downloaded from the Arabidopsis Information Resource (https://phytozome.jgi.doe.gov/pz/portal.html) and the National Center for Biotechnology Information server (NCBI; http://www.ncbi.nlm.nih.gov/). Transit peptides were predicted by ChloroP (http://www.cbs.dtu.dk/services/ChloroP/) (Emanuelsson et al., 1999). Structural data was obtained from the PDB homepage (https://www.rcsb.org/) and processed with ChimeraX (https://www.cgl.ucsf.edu/chimerax/) (Pettersen et al., 2021). Multiple sequence alignments were generated with the CLC workbench software (v8.1) and protein features were visualized with Protter (https://wlab.ethz.ch/protter/start/) (Omasits et al., 2014). Co-migration of stromal proteins was

examined

with

the

online tool

PCom-DB

543 (http://pcomdb.lowtem.hokudai.ac.jp/proteins/top) (Takabayashi et al., 2017). Boxplots were drawn with BoxPlotR (http://shiny.chemgrid.org/boxplotr/) (Spitzer et al., 2014).

\section{Plant Material and Growth Conditions}

T-DNA lines for atcgl160-1 (SALK_057229, Col-0 background), atcgld11-1 (SALK_019326C,

548 Col-0 background) and atalb4-1 (SALK_136199C) were obtained from the SALK collection

549 (Alonso et al., 2003). Plants were grown on potting soil (A210, Stender, Schermbeck,

550 Germany) under controlled greenhouse conditions $\left(70-90 \mu \mathrm{mol}\right.$ photons $\mathrm{m}^{-2} \mathrm{~s}^{-1}, 16 / 8 \mathrm{~h}$ light/dark cycles), or in climate chambers on an $8 \mathrm{~h}$ light/16h dark cycle for biochemical and physiological analyses. Fertilizer was added to plants grown under greenhouse conditions according to the manufacturer's recommendations (Osmocote Plus; $15 \%$ nitrogen [w/v], 11\% [w/v] $\mathrm{P}_{2} \mathrm{O}_{5}, 13 \%[\mathrm{w} / \mathrm{v}] \mathrm{K}_{2} \mathrm{O}$, and 2\% [w/v] MgO; Scotts, Germany). For domain-specific complementation assays, either the complete coding region of AtCGL160 ( $P_{355}$ :AtCGL160) or parts of the CDS corresponding to amino acids 1-206 (P35s:AtCGL160N) and 207-350 ( $P_{35 s: A t C G L 160 C)}$ were cloned into the binary Gateway vector pB2GW7 (Karimi et al., 2002), placing the genes under control of the 35S CaMV promoter. The transit peptide coding sequence (for amino acids 1-46) was fused to the AtCGL160C CDS in the case of the $P_{35 s}$ :AtCGL160C vector. The constructs were first transformed into Agrobacterium tumefaciens strain GV3101, and then into atcgl160-1 plants by the floral-dip method (Clough and Bent, 1998). T1 plants were selected by screening for Basta resistance. Basta positives were screened for equal amounts of the AtCGL160 transcript by RNA gel-blot hybridization as described below. 
$\underline{\text { Transmission electron microscopy }}$

567

568

569

570

571

572

573

574

575

576

577

578

579

580

581

582

583

584

585

586

587

588

589

590

591

592

593

594

595

596

597

Leaf pieces of about $1.5 \times 1.0 \mathrm{~mm}$ were cut with a new double edge razor blade (Feather, Osaka, Japan) and immediately immersed in fixation buffer $(0.1 \mathrm{M}$ sodium phosphate buffer, $\mathrm{pH} 7.4,2.5 \%[\mathrm{v} / \mathrm{v}]$ glutaraldehyde, $4 \%[\mathrm{v} / \mathrm{v}]$ formaldehyde) at room temperature. A mild vacuum (about 20 mbar) was applied until the leaf pieces did sink, the fixation buffer replaced with fresh one and the samples fixed overnight at $4{ }^{\circ} \mathrm{C}$. After three 10-min washes in sodium phosphate buffer $(\mathrm{pH} 7.4)$, the samples were osmicated with $1 \%$ osmium tetroxide and $1.5 \%$ potassium ferricyanide in $0.1 \mathrm{M}$ sodium phosphate buffer $\left(\mathrm{pH} \mathrm{7.4)}\right.$ for $60 \mathrm{~min}$ at $4^{\circ} \mathrm{C}$. The samples were rinsed three times for 10 min each in distilled water and incubated in 1\% uranyl acetate (in distilled water) at $4^{\circ} \mathrm{C}$ overnight. After three washes of 10 min each in distilled water the samples were dehydrated using increasing concentrations of ethanol and infiltrated, with propylene oxide as intermediate solvent, in glycid ether 100 (formerly Epon 812; Serva, Heidelberg, Germany) following standard procedures. Polymerization was carried out for 40 $48 \mathrm{~h}$ at $65{ }^{\circ} \mathrm{C}$. Ultrathin sections $(\sim 60 \mathrm{~nm})$ were cut with a diamond knife (type ultra $35^{\circ}$; Diatome, Biel, Suisse) on an EM UC7 ultramicrotome (Leica Microsystems, Wetzlar, Germany) and mounted on single-slot Pioloform-coated copper grids (Plano, Wetzlar, Germany). The sections were stained using uranylacetate and lead citrate (Reynolds, 1963) and viewed with a JEM-1400 Plus transmission electron microscope (JEOL, Tokyo, Japan) operated at 80kV. Micrographs were taken using a 3.296 × 2.472 pixels charge-coupled device camera (Ruby, JEOL).

\section{$\underline{\text { Chl a Fluorescence Measurements }}$}

In vivo $\mathrm{Chl}$ a fluorescence of whole plants was measured using an imaging $\mathrm{Chl}$ fluorometer (Imaging PAM, Walz, Effeltrich, Germany). Plants were dark-adapted for 20 min and exposed to a pulsed, blue measuring beam ( $4 \mathrm{~Hz}$, intensity 3 , gain 3 , damping 2 ; $F_{O}$ ) and a saturating light flash (intensity 10 ) to calculate $\mathrm{F}_{\mathrm{v}} / \mathrm{F}_{\mathrm{M}}$. If not indicated otherwise, transient $N P Q$ induction was measured at $145 \mu \mathrm{mol}$ photons $\mathrm{m}^{-2} \mathrm{~s}^{-1}$.

\section{ECS Measurements}

ECS measurements were performed using the Dual-PAM-100 (Walz, Effeltrich, Germany) equipped with a P515/535 emitter-detector module (Schreiber and Klughammer, 2008). The measurement was carried out at $23^{\circ} \mathrm{C}$ under ambient $\mathrm{CO}_{2}$ conditions. Plants grown in short- 
day conditions for four weeks were light-adapted, and detached leaves were illuminated for at least $10 \mathrm{~min}$ with $129 \mu \mathrm{mol}$ photons $\mathrm{m}^{-2} \mathrm{~s}^{-1}$ red light. After illumination, dark-interval relaxation kinetics (DIRK) were measured in the ms to $s$ range. Values for pmf (ECSt), and proton conductivity $\left(\mathrm{gH}^{+}\right)$were calculated as described (Cruz et al., 2001; Schreiber and Klughammer, 2008). Briefly, the maximum amplitude of the inverse electrochromic band-shift kinetic was measured in the second range, and normalized to a single saturating P515 pulse measured after 4 minutes of dark incubation. For proton conductivity, electrochromic band-shift kinetics were recorded in the millisecond range for 5 consecutive periods of $2 \mathrm{sec}$, separated by dark intervals of $30 \mathrm{sec}$. The combined signals were fitted to a single exponential decay function and the reciprocal value of the lifetime was used to estimate the proton conductivity (Kanazawa and Kramer, 2002).

\section{AtCGL160 Antibody Generation}

Rabbit antibodies were generated against AtCGL160 that had been heterologously expressed in Escherichia coli, and then purified. To this end, the coding sequence corresponding to AtCGL160 $29-206$ was cloned into the pMal-c5x vector (New England Biolabs) and purified on amylose columns (New England Biolabs) according to the manufacturer's instructions. The protein was injected into rabbits for antibody production (Pineda, Berlin, Germany). To reduce epitope cross-reactions, the antiserum was purified on a column crosslinked with heterologously expressed AtCGL160 $29-206$ fused to the glutathione-S-transferase (GST) tag. Purified antibody was employed at a dilution of 1:1000. Signals were detected by enhanced chemiluminescence (Pierce ${ }^{\mathrm{TM}}$ ECL Western Blotting Substrate, Thermo Scientific) using an ECL reader system (Fusion FX7; PeqLab, Erlangen, Germany).

\section{Nucleic Acid Analysis}

Total RNA from snap-frozen leaves was extracted with the RNeasy Plant Mini Kit (Qiagen) according to the supplier's instructions. Samples equivalent to 8 or $20 \mu \mathrm{g}$ total RNA were fractionated by electrophoresis in formaldehyde-containing agarose gels (1.2\%), blotted onto nylon membranes (Hybond-N+, Amersham Bioscience) and fixed by UV radiation (Stratalinker® UV Crosslinker 1800). To control for equal loading, abundant RNAs on nylon membranes were stained with methylene blue solution $(0.02 \%[\mathrm{w} / \mathrm{v}]$ methylene blue, $0.3 \mathrm{M}$ sodium acetate $\mathrm{pH}$ 5.5). To detect gene-specific transcripts, DNA fragments amplified from cDNA were labelled with radioactive $\left[\alpha^{-32} \mathrm{P}\right] \mathrm{dCTP}$ and subsequently used as probes in hybridization experiments (see Supplemental Table S2 for primer information). Signals were detected with the Typhoon Phosphor Imager System (GE Healthcare). 


\section{$\underline{\text { Protein Analysis }}$}

635

636

637

638

639

640

641

642

643

644

645

646

647

648

649

650

651

652

653

654

655

656

657

658

659

660

661

662

663

664

665

666

667

668

Leaves from 4-week-old plants grown under short-day conditions were harvested shortly after the onset of the light period, and thylakoid membrane-enriched samples were isolated according to Rühle et al. (2014). Crosslinking of thylakoids was performed by incubation with $2.5 \mathrm{mM}$ dithiobis(succinimidyl propionate) (DSP, Thermo Scientific). After incubation for $20 \mathrm{~min}$ on ice, crosslinking was quenched with $60 \mathrm{mM}$ Tris/ $\mathrm{HCl}(\mathrm{pH} 7.5)$. Chl concentrations were determined as described in Porra et al. (1989). For immunotitrations, thylakoid membrane pellets were resuspended in loading buffer $(100 \mathrm{mM}$ Tris/ $\mathrm{HCl} \mathrm{pH} \mathrm{6.8,50} \mathrm{mM} \mathrm{dithiothreitol}$ [DTT], $8 \%$ [w/v] SDS, 24\% [w/v] glycerol and 0.02\% [w/v] bromophenol blue). Denaturation for $5 \mathrm{~min}$ at $70^{\circ} \mathrm{C}$ and protein fractionation on Tricine-SDS-PAGE gels (10\% gels supplemented with 4M Urea) was carried out according to Schägger (2006). Immunodetections were performed as described below. Sample preparation for BN-PAGE was performed with freshly prepared thylakoids as described in Peng et al. (2008). First, membranes were washed twice in wash buffer (20\% glycerol, $25 \mathrm{mM}$ BisTris/HCl pH 7.0). Then, samples were treated with wash buffer containing $1 \%(\mathrm{w} / \mathrm{v}) \mathrm{n}$-dodecyl $\beta$-D-maltoside and adjusted to $1 \mathrm{ml} \mathrm{mg}^{-1} \mathrm{Chl}$ for 10 min on ice. After centrifugation $\left(16,000 \mathrm{~g}, 20 \mathrm{~min}, 4^{\circ} \mathrm{C}\right)$, supernatants were supplemented with $1 / 10$ volume of $\mathrm{BN}$ sample buffer $(100 \mathrm{mM}$ BisTris/HCl pH 7.0, $750 \mathrm{mM} \varepsilon$-aminocaproic acid, $5 \%$ [w/v] Coomassie G-250). BN-PAGE gels (4-12\% gradient) were prepared as described in Schägger et al. (1994). Solubilized samples corresponding to $60 \mu \mathrm{g} C \mathrm{Cl}$ were loaded per lane and gels were run at $4^{\circ} \mathrm{C}$ overnight. To separate complexes into their subunits, BN-PAGE strips were treated with denaturing buffer $\left(0.2 \mathrm{M} \mathrm{Na}_{2} \mathrm{CO}_{3}, 5 \%\right.$ [w/v] SDS, $50 \mathrm{mM}$ DTT) for 30 min at room temperature and loaded on Tricine-SDS-PAGE gels. Gels were subsequently subjected to immunoblot analysis with antibodies against $\mathrm{CF}_{1}-\mathrm{CF}_{\mathrm{O}}$ subunits and AtCGL160, as described below.

For analysis of the stromal $\mathrm{CF}_{1}$ intermediate, intact chloroplasts from 4-week-old plants were isolated according to Rühle et al. (2021). After lysis in $25 \mathrm{mM} \mathrm{HEPES/KOH} \mathrm{(pH} \mathrm{7.5)} \mathrm{containing}$ $5 \mathrm{mM} \mathrm{MgCl} 2$ for $30 \mathrm{~min}$ on ice, the stromal fraction was separated from membranes by centrifugation at $35,000 \mathrm{~g}$ for $30 \mathrm{~min}\left(4^{\circ} \mathrm{C}\right)$. Protein concentration was measured using the Bradford Protein Assay (Bio-Rad). Stromal BN analysis was performed according to Reiter et al. (2020). In brief, chloroplast-enriched pellets were resuspended in BN washing buffer and mechanically disrupted by passage through an $0.45-\mathrm{mm}$ syringe. The stromal fraction was separated from membranes by centrifugation at $35,000 \mathrm{~g}$ for $30 \mathrm{~min}$ (at $4^{\circ} \mathrm{C}$ ). $100 \mu \mathrm{g}$ of total soluble protein was mixed with 1/10 volume of BN sample buffer before fractionation in the first dimension as described above. 
Proteins fractionated by gel electrophoresis were transferred to polyvinylidene difluoride membranes (PVDF) (Immobilon®-P, Millipore) using a semi-dry blotting system (Biorad) as described in the supplier's instructions. After blocking with TBST $(10 \mathrm{mM}$ Tris/HCl pH 8.0, 150 $\mathrm{mM} \mathrm{NaCl}$ and $0.1 \%[\mathrm{v} / \mathrm{v}]$ Tween-20) supplemented with 3\% (w/v) skim milk powder, the membranes were incubated with antibodies at $4^{\circ} \mathrm{C}$ overnight. Antibodies used in this study were obtained from Agrisera ( $\mathrm{CF}_{1-} \beta$ : AS05 085, 1:5000; $\mathrm{CF}_{1-\gamma}$ : AS08 312, 1:5000; CFo-b: AS10 1604, 1:5000; CFo-C: AS09 591, 1:3000; and AtCGL160: AS12 1853, 1:1000).

Yeast-two-Hybrid Experiments

Yeast two-hybrid assays were carried out using the Matchmaker Two-Hybrid System Kit (Clontech). The AtCGL160 CDS without the signal peptide (see Supplemental Table S2 for primer information) was cloned into the bait vector pGBKT7 (BD-AtCGL160), whereas the coding sequences of $C F_{1}-\alpha,-\beta,-\gamma,-\delta,-\varepsilon$, the soluble domains of $C_{O}-b$ (51-184 aa) and $b^{\prime}$ (109-219 aa), AtCGL160 and the CF $_{1}$ assembly factor AtCGLD11 were cloned into the prey vector pGADT7 (named AD-CF $1-\alpha, A D-C F_{1}-\beta, A D-C_{1}-\gamma, A D-C F_{1}-\delta, A D-C F_{1-\varepsilon}, A D-C F_{O}-b, A D-$

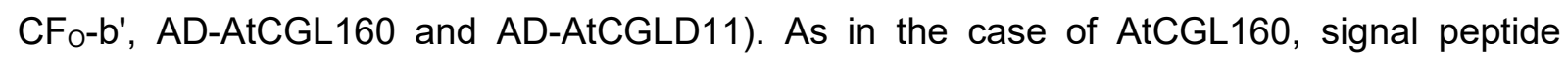
sequences were omitted from the nucleus-encoded subunits $C F_{1-\gamma}, C F_{1}-\delta, C F_{O}-b^{\prime}$ and AtCGLD11. For binding-domain analysis of $C_{1-} \beta$, the respective CDS was sub-divided into three parts, according to Groth and Pohl (2001) and cloned into pGADT7. In the case of AtCGL160N binding-site analysis, sequences coding for 29-74, 75-105, 106-134, 135-160 and 161-206 aa were deleted from the BD-AtCGL160 vector using a site-directed mutagenesis kit (NEB). Primers are listed in Supplemental Table S2. Bait and prey vectors were cotransformed into AH109 yeast strains (Clontech) following manufacturer's instructions. Cotransformants were selected on synthetic dropout (SD) medium (Clontech) lacking leucine and tryptophan (-LT). In order to identify protein interactions, double transformants were grown on SD medium lacking leucine, tryptophan, histidine, and adenine (-LTHA).

\section{Co-immunoprecipitation}

Freshly extracted thylakoids corresponding to $\sim 10 \mathrm{mg}$ chlorophyll were resuspended in $500 \mu \mathrm{l}$ extraction buffer $(50 \mathrm{mM}$ Tris/ $\mathrm{HCl} \mathrm{pH} 7.5,150 \mathrm{mM} \mathrm{NaCl}, 1 \mathrm{mM} \mathrm{MgCl} 2,5 \%$ [w/v] glycerol, $1 \%$ [v/v] Nonidet P40 [NP40], 0.2 mM phenylmethylsulfonyl fluoride [PMSF]) and solubilized for 30 min on ice. After centrifugation at $35,000 \mathrm{~g}$ for $30 \mathrm{~min}$ and $4^{\circ} \mathrm{C}$, the supernatant was added to $20 \mu \mathrm{l}$ Dynabeads (Thermo Scientific), equilibrated with equilibration buffer (50 mM Tris/ $\mathrm{HCl} \mathrm{pH}$ 
according to the manufacturer's instructions. The suspension was incubated with rotation for $3 \mathrm{~h}$ at $4^{\circ} \mathrm{C}$, washed three times with equilibration buffer, and twice with the same buffer but omitting NP40. Proteins were eluted with $100 \mu \mathrm{l} 0.1 \mathrm{M}$ glycine $\mathrm{pH} 2.0$ for $10 \mathrm{~min}$ and neutralized with $100 \mu \mathrm{l} 0.1 \mathrm{M}$ ammonium bicarbonate. After treatment with $10 \mu \mathrm{l}$ of $45 \mathrm{mM}$ DTT and $10 \mu \mathrm{l}$ of $0.1 \mathrm{M}$ iodoacetamide, samples were digested with $1.5 \mu \mathrm{g}$ of trypsin at $37^{\circ} \mathrm{C}$ overnight. Peptides were desalted with home-made C18 stage tips (Rappsilber et al., 2003), vacuumdried to near dryness and stored at $-80^{\circ} \mathrm{C}$. LC MS/MS run and data analysis were performed as described in Reiter et al. (2020).

\section{Author Contributions}

B.R. and T.R. designed research. B.R., L.R., G.M., S.G. and T.R. carried out experiments. B.R., D.L. and T.R. prepared the article. T.R. supervised the whole study.

\section{Acknowledgments}

We thank Tim Scheibenbogen, Michael Berger and Tanja Neufeld for technical assistance with Yeast-two-hybrid experiments, Tim Dreißig for technical assistance with heterologous expression of AtCGL160N, and Paul Hardy for critical comments on the manuscript.

\section{Funding information}

This work was funded by the German Science Foundation (DFG, Research Unit FOR2092, project number 239484859, grant GE 1110/9-1 for S.G. and RU 1945/2-1 for T.R.) 


\section{References}

Alonso, J.M., Stepanova, A.N., Leisse, T.J., Kim, C.J., Chen, H., Shinn, P., Stevenson, D.K., Zimmerman, J., Barajas, P., Cheuk, R., Gadrinab, C., Heller, C., Jeske, A., Koesema, E., Meyers, C.C., Parker, H., Prednis, L., Ansari, Y., Choy, N., Deen, H., Geralt, M., Hazari, N., Hom, E., Karnes, M., Mulholland, C., Ndubaku, R., Schmidt, I., Guzman, P., Aguilar-Henonin, L., Schmid, M., Weigel, D., Carter, D.E., Marchand, T., Risseeuw, E., Brogden, D., Zeko, A., Crosby, W.L., Berry, C.C., and Ecker, J.R. (2003). Genome-wide insertional mutagenesis of Arabidopsis thaliana. Science (New York, N.Y.) 301, 653-657.

Avenson, T.J., Kanazawa, A., Cruz, J.A., Takizawa, K., Ettinger, W.E., and Kramer, D.M. (2005). Integrating the proton circuit into photosynthesis: progress and challenges. Plant Cell and Environment 28, 97-109.

Bedard, J., Trosch, R., Wu, F.J., Ling, Q.H., Flores-Perez, U., Topel, M., Nawaz, F., and Jarvisa, P. (2017). Suppressors of the Chloroplast Protein Import Mutant tic40 Reveal a Genetic Link between Protein Import and Thylakoid Biogenesis. Plant Cell 29, 1726-+.

Benz, M., Bals, T., Gügel, I.L., Piotrowski, M., Kuhn, A., Schünemann, D., Soll, J., and Ankele, E. (2009). Alb4 of Arabidopsis promotes assembly and stabilization of a non chlorophyll-binding photosynthetic complex, the CF1CF0-ATP synthase. Molecular plant 2, 1410-1424.

Birkenhäger, R., Greie, J.C., Altendorf, K., and Deckers-Hebestreit, G. (1999). F0 complex of the Escherichia coli ATP synthase. Not all monomers of the subunit $\mathrm{C}$ oligomer are involved in F1 interaction. European journal of biochemistry / FEBS 264, 385-396.

Cabezon, E., Montgomery, M.G., Leslie, A.G., and Walker, J.E. (2003). The structure of bovine F1-ATPase in complex with its regulatory protein IF1. Nat Struct Biol 10, 744750.

Chen, G.G., and Jagendorf, a.T. (1994). Chloroplast molecular chaperone-assisted refolding and reconstitution of an active multisubunit coupling factor CF1 core. Proceedings of the National Academy of Sciences of the United States of America 91, 11497-11501.

Clough, S.J., and Bent, A.F. (1998). Floral dip: a simplified method for Agrobacteriummediated transformation of Arabidopsis thaliana. Plant J 16, 735-743.

Cruz, J.A., Sacksteder, C.A., Kanazawa, A., and Kramer, D.M. (2001). Contribution of electric field (Delta psi) to steady-state transthylakoid proton motive force (pmf) in vitro and in vivo. control of pmf parsing into Delta psi and Delta $\mathrm{pH}$ by ionic strength. Biochemistry 40, 1226-1237. 
Daum, B., Nicastro, D., Austin, J., Mclntosh, J.R., and Kühlbrandt, W. (2010). Arrangement of photosystem II and ATP synthase in chloroplast membranes of spinach and pea. The Plant cell 22, 1299-1312.

Deckers-Hebestreit, G. (2013). Assembly of the Escherichia coli FoF1 ATP synthase involves distinct subcomplex formation. Biochemical Society transactions 41, 12881293.

Eberhard, S., Loiselay, C., Drapier, D., Bujaldon, S., Girard-Bascou, J., Kuras, R., Choquet, Y., and Wollman, F.-A. (2011). Dual functions of the nucleus-encoded factor TDA1 in trapping and translation activation of atpA transcripts in Chlamydomonas reinhardtii chloroplasts. The Plant journal : for cell and molecular biology 67, 1055-1066.

Emanuelsson, O., Nielsen, H., and von Heijne, G. (1999). ChloroP, a neural networkbased method for predicting chloroplast transit peptides and their cleavage sites. Protein Science : A Publication of the Protein Society 8, 978-984.

Franklin, M.J., Brusilow, W.S.a., and Woodbury, D.J. (2004). Determination of proton flux and conductance at $\mathrm{pH} 6.8$ through single FO sectors from Escherichia coli. Biophysical journal 87, 3594-3599.

Fristedt, R., Martins, N.F., Strenkert, D., Clarke, C.A., Suchoszek, M., Thiele, W., Schöttler, M.A., and Merchant, S.S. (2015). The thylakoid membrane protein CGL160 supports CF1CF0 ATP synthase accumulation in Arabidopsis thaliana. PloS one 10, e0121658-e0121658.

Gay, N.J. (1984). Construction and characterization of an Escherichia coli strain with a uncl mutation. Journal of bacteriology 158, 820-825.

Grahl, S., Reiter, B., Gügel, Irene L., Vamvaka, E., Gandini, C., Jahns, P., Soll, J., Leister, D., and Rühle, T. (2016). The Arabidopsis Protein CGLD11 Is Required for Chloroplast ATP Synthase Accumulation. Molecular Plant 9, 885-899.

Groth, G., and Pohl, E. (2001). The structure of the chloroplast F1-ATPase at 3.2 A resolution. The Journal of biological chemistry 276, 1345-1352.

Hahn, A., Vonck, J., Mills, D.J., Meier, T., and Kühlbrandt, W. (2018). Structure, mechanism, and regulation of the chloroplast ATP synthase. Science (New York, N.Y.) 360, eaat4318-eaat4318.

He, J., Ford, H.C., Carroll, J., Douglas, C., Gonzales, E., Ding, S., Fearnley, I.M., and Walker, J.E. (2018). Assembly of the membrane domain of ATP synthase in human mitochondria. Proceedings of the National Academy of Sciences, 201722086201722086.

Hilbers, F., Eggers, R., Pradela, K., Friedrich, K., Herkenhoff-Hesselmann, B., Becker, E., and Deckers-Hebestreit, G. (2013). Subunit $\delta$ is the key player for assembly of 
the $\mathrm{H}(+)$-translocating unit of Escherichia coli $F(O) F 1$ ATP synthase. The Journal of biological chemistry 288, 25880-25894.

Junge, W., and Nelson, N. (2015). ATP synthase. Annual Review of Biochemistry 84, 631657.

Kanazawa, A., and Kramer, D.M. (2002). In vivo modulation of nonphotochemical exciton quenching (NPQ) by regulation of the chloroplast ATP synthase. Proceedings of the National Academy of Sciences of the United States of America 99, 12789-12794.

Kanazawa, A., Ostendorf, E., Kohzuma, K., Hoh, D., Strand, D.D., Sato-Cruz, M., Savage, L., Cruz, J.A., Fisher, N., Froehlich, J.E., and Kramer, D.M. (2017). Chloroplast ATP Synthase Modulation of the Thylakoid Proton Motive Force: Implications for Photosystem I and Photosystem II Photoprotection. Frontiers in Plant Science 8, 1-12.

Karimi, M., Inze, D., and Depicker, A. (2002). GATEWAY vectors for Agrobacteriummediated plant transformation. Trends Plant Sci 7, 193-195.

Karpowicz, S.J., Prochnik, S.E., Grossman, a.R., and Merchant, S.S. (2011). The GreenCut2 Resource, a Phylogenomically Derived Inventory of Proteins Specific to the Plant Lineage. Journal of Biological Chemistry 286, 21427-21439.

Kohzuma, K., Froehlich, J.E., Davis, G.A., Temple, J.A., Minhas, D., Dhingra, A., Cruz, J.A., and Kramer, D.M. (2017). The role of light-dark regulation of the chloroplast ATP synthase. Frontiers in Plant Science 8, 1-14.

Liu, J., Hicks, D.B., and Krulwich, T.a. (2013). Roles of Atpl and two YidC-type proteins from alkaliphilic Bacillus pseudofirmus OF4 in ATP synthase assembly and nonfermentative growth. Journal of bacteriology 195, 220-230.

Mao, J., Chi, W., Ouyang, M., He, B., Chen, F., and Zhang, L. (2015). PAB is an assembly chaperone that functions downstream of chaperonin 60 in the assembly of chloroplast ATP synthase coupling factor 1. Proceedings of the National Academy of Sciences 112, 4152-4157.

Merchant, S.S., Prochnik, S.E., Vallon, O., Harris, E.H., Karpowicz, S.J., Witman, G.B., Terry, A., Salamov, A., Fritz-Laylin, L.K., Maréchal-Drouard, L., Marshall, W.F., Qu, L.-H., Nelson, D.R., Sanderfoot, A.a., Spalding, M.H., Kapitonov, V.V., Ren, Q., Ferris, P., Lindquist, E., Shapiro, H., Lucas, S.M., Grimwood, J., Schmutz, J., Cardol, P., Cerutti, H., Chanfreau, G., Chen, C.-L., Cognat, V., Croft, M.T., Dent, R., Dutcher, S., Fernández, E., Fukuzawa, H., González-Ballester, D., GonzálezHalphen, D., Hallmann, A., Hanikenne, M., Hippler, M., Inwood, W., Jabbari, K., Kalanon, M., Kuras, R., Lefebvre, P.a., Lemaire, S.D., Lobanov, A.V., Lohr, M., Manuell, A., Meier, I., Mets, L., Mittag, M., Mittelmeier, T., Moroney, J.V., Moseley, J., Napoli, C., Nedelcu, A.M., Niyogi, K., Novoselov, S.V., Paulsen, I.T., 
Pazour, G., Purton, S., Ral, J.-P., Riaño-Pachón, D.M., Riekhof, W., Rymarquis, L., Schroda, M., Stern, D., Umen, J., Willows, R., Wilson, N., Zimmer, S.L., Allmer, J., Balk, J., Bisova, K., Chen, C.-J., Elias, M., Gendler, K., Hauser, C., Lamb, M.R., Ledford, H., Long, J.C., Minagawa, J., Page, M.D., Pan, J., Pootakham, W., Roje, S., Rose, A., Stahlberg, E., Terauchi, A.M., Yang, P., Ball, S., Bowler, C., Dieckmann, C.L., Gladyshev, V.N., Green, P., Jorgensen, R., Mayfield, S., Mueller-Roeber, B., Rajamani, S., Sayre, R.T., Brokstein, P., Dubchak, I., Goodstein, D., Hornick, L., Huang, Y.W., Jhaveri, J., Luo, Y., Martínez, D., Ngau, W.C.A., Otillar, B., Poliakov, A., Porter, A., Szajkowski, L., Werner, G., Zhou, K., Grigoriev, I.V., Rokhsar, D.S., and Grossman, A.R. (2007). The Chlamydomonas genome reveals the evolution of key animal and plant functions. Science (New York, N.Y.) 318, 245-250.

Murphy, B.J., Klusch, N., Langer, J., Mills, D.J., Yildiz, O., and Kuhlbrandt, W. (2019). Rotary substates of mitochondrial ATP synthase reveal the basis of flexible F1-Fo coupling. Science 364.

Naumenko, N., Morgenstern, M., Rucktaschel, R., Warscheid, B., and Rehling, P. (2017). INA complex liaises the F1Fo-ATP synthase membrane motor modules. Nat Commun 8, 1237.

Obayashi, T., Hayashi, S., Saeki, M., Ohta, H., and Kinoshita, K. (2009). ATTED-II provides coexpressed gene networks for Arabidopsis. Nucleic acids research 37, D987-991.

Omasits, U., Ahrens, C.H., Müller, S., and Wollscheid, B. (2014). Protter: Interactive protein feature visualization and integration with experimental proteomic data. Bioinformatics 30, 884-886.

Ort, D.R., and Oxborough, K. (1992). In Situ Regulation of Chloroplast Coupling Factor Activity. Annual Review of Plant Physiology and Plant Molecular Biology 43, 269-291.

Ozaki, Y., Suzuki, T., Kuruma, Y., Ueda, T., and Yoshida, M. (2008). Uncl protein can mediate ring-assembly of c-subunits of FoF1-ATP synthase in vitro. Biochemical and biophysical research communications 367, 663-666.

Peng, L., Shimizu, H., and Shikanai, T. (2008). The chloroplast NAD(P)H dehydrogenase complex interacts with photosystem I in Arabidopsis. The Journal of biological chemistry 283, 34873-34879.

Pettersen, E.F., Goddard, T.D., Huang, C.C., Meng, E.C., Couch, G.S., Croll, T.I., Morris, J.H., and Ferrin, T.E. (2021). UCSF ChimeraX: Structure visualization for researchers, educators, and developers. Protein Science 30, 70-82. 
Pfalz, J., Bayraktar, O.A., Prikryl, J., and Barkan, A. (2009). Site-specific binding of a PPR protein defines and stabilizes $5^{\prime}$ and $3^{\prime}$ 'mRNA termini in chloroplasts. The EMBO journal 28, 2042-2052.

Porra, R.J., Thompson, W.A., and Kriedemann, P.E. (1989). Determination of accurate extinction coefficients and simultaneous equations for assaying chlorophylls $a$ and $b$ extracted with four different solvents: verification of the concentration of chlorophyll standards by atomic absorption spectroscopy. Biochim Biophys Acta 975, 384-394.

Rappsilber, J., Ishihama, Y., and Mann, M. (2003). Stop and go extraction tips for matrixassisted laser desorption/ionization, nanoelectrospray, and LC/MS sample pretreatment in proteomics. Anal Chem 75, 663-670.

Reiland, S., Messerli, G., Baerenfaller, K., Gerrits, B., Endler, A., Grossmann, J., Gruissem, W., and Baginsky, S. (2009). Large-scale Arabidopsis phosphoproteome profiling reveals novel chloroplast kinase substrates and phosphorylation networks. Plant physiology 150, 889-903.

Reiland, S., Finazzi, G., Endler, A., Willig, A., Baerenfaller, K., Grossmann, J., Gerrits, B., Rutishauser, D., Gruissem, W., Rochaix, J.-D., and Baginsky, S. (2011). Comparative phosphoproteome profiling reveals a function of the STN8 kinase in fine-tuning of cyclic electron flow (CEF). Proceedings of the National Academy of Sciences of the United States of America 108, 12955-12960.

Reiter, B., Vamvaka, E., Marino, G., Kleine, T., Jahns, P., Bolle, C., Leister, D., and Rühle, T. (2020). The Arabidopsis Protein CGL20 Is Required for Plastid 50S Ribosome Biogenesis. Plant physiology 182, 1222-1238.

Reynolds, E.S. (1963). Use of Lead Citrate at High Ph as an Electron-Opaque Stain in Electron Microscopy. Journal of Cell Biology 17, 208-\&.

Robinson, G.C., Bason, J.V., Montgomery, M.G., Fearnley, I.M., Mueller, D.M., Leslie, A.G., and Walker, J.E. (2013). The structure of F(1)-ATPase from Saccharomyces cerevisiae inhibited by its regulatory protein IF(1). Open Biol 3, 120164.

Roitinger, E., Hofer, M., Kocher, T., Pichler, P., Novatchkova, M., Yang, J., Schlogelhofer, P., and Mechtler, K. (2015). Quantitative phosphoproteomics of the ataxia telangiectasia-mutated (ATM) and ataxia telangiectasia-mutated and rad3related (ATR) dependent DNA damage response in Arabidopsis thaliana. Mol Cell Proteomics 14, 556-571.

Rühle, T., and Leister, D. (2015). Assembly of F1F0-ATP synthases. Biochimica et Biophysica Acta (BBA) - Bioenergetics 1847, 849-860.

Rühle, T., Dann, M., Reiter, B., Schünemann, D., Naranjo, B., Penzler, J.-f., Kleine, T., and Leister, D. (2021). PGRL2 triggers degradation of PGR5 in the absence of PGRL1. Nature communications 12, 3941-3941. 
Rühle, T., Razeghi, J.A., Vamvaka, E., Viola, S., Gandini, C., Kleine, T., Schünemann, D., Barbato, R., Jahns, P., and Leister, D. (2014). The Arabidopsis protein CONSERVED ONLY IN THE GREEN LINEAGE160 promotes the assembly of the membranous part of the chloroplast ATP synthase. Plant physiology 1. Wittenb, 207226.

Schägger, H. (2006). Tricine-SDS-PAGE. Nature protocols 1, 16-22.

Schöttler, M.A., Tóth, S.Z., Boulouis, A., and Kahlau, S. (2014). Photosynthetic complex stoichiometry dynamics in higher plants: biogenesis, function, and turnover of ATP synthase and the cytochrome $b 6 \mathrm{f}$ complex. Journal of experimental botany.

Schreiber, U., and Klughammer, C. (2008). New accessory for the Dual-PAM-100 : The P515 / 535 module and examples of its application. PAM Application Notes 10, 1-10.

Sobti, M., Smits, C., Wong, A.S., Ishmukhametov, R., Stock, D., Sandin, S., and Stewart, A.G. (2016). Cryo-EM structures of the autoinhibited E. coli ATP synthase in three rotational states. Elife 5, 1-18.

Song, J., Pfanner, N., and Becker, T. (2018). Assembling the mitochondrial ATP synthase. Proceedings of the National Academy of Sciences of the United States of America $115,2850-2852$.

Spitzer, M., Wildenhain, J., Rappsilber, J., and Tyers, M. (2014). BoxPlotR: a web tool for generation of box plots. Nat Methods 11, 121-122.

Suzuki, T., Ozaki, Y., Sone, N., Feniouk, B.a., and Yoshida, M. (2007). The product of uncl gene in F1Fo-ATP synthase operon plays a chaperone-like role to assist c-ring assembly. Proceedings of the National Academy of Sciences of the United States of America 104, 20776-20781.

Takabayashi, A., Takabayashi, S., Takahashi, K., Watanabe, M., Uchida, H., Murakami, A., Fujita, T., Ikeuchi, M., and Tanaka, A. (2017). PCoM-DB update: A protein comigration database for photosynthetic organisms. Plant and Cell Physiology 58, e10e10.

Tanigawara, M., Tabata, K.V., Ito, Y., Ito, J., Watanabe, R., Ueno, H., Ikeguchi, M., and Noji, H. (2012). Role of the DELSEED loop in torque transmission of F1-ATPase. Biophys J 103, 970-978.

Tomizioli, M., Lazar, C., Brugiere, S., Burger, T., Salvi, D., Gatto, L., Moyet, L., Breckels, L.M., Hesse, A.M., Lilley, K.S., Seigneurin-Berny, D., Finazzi, G., Rolland, N., and Ferro, M. (2014). Deciphering Thylakoid Sub-compartments using a Mass Spectrometry-based Approach. Molecular \& Cellular Proteomics 13, 2147-2167.

Trosch, R., Topel, M., Flores-Perez, U., and Jarvis, P. (2015). Genetic and Physical Interaction Studies Reveal Functional Similarities between ALBINO3 and ALBINO4 in Arabidopsis. Plant physiology 169, 1292-1306. 
Vollmar, M., Schlieper, D., Winn, M., Büchner, C., and Groth, G. (2009). Structure of the c14 rotor ring of the proton translocating chloroplast ATP synthase. The Journal of biological chemistry 284, 18228-18235.

von Ballmoos, C., Wiedenmann, A., and Dimroth, P. (2009). Essentials for ATP synthesis by F1F0 ATP synthases. Annual review of biochemistry 78, 649-672.

Yap, A., Kindgren, P., Colas Des Francs-Small, C., Kazama, T., Tanz, S.K., Toriyama, K., and Small, I. (2015). AEF1/MPR25 is implicated in RNA editing of plastid atpF and mitochondrial nad5, and also promotes atpF splicing in Arabidopsis and rice. Plant Journal 81, 661-669.

Zhang, L., Rochaix, J.D., and Peng, L. (2020). Regulation of the biogenesis of chloroplast ATP synthase. Advances in Botanical Research 96, 205-228.

Zhang, L., Duan, Z., Zhang, J., and Peng, L. (2016). BIOGENESIS FACTOR REQUIRED FOR ATP SYNTHASE 3 Facilitates Assembly of the Chloroplast ATP Synthase Complex in Arabidopsis. Plant Physiology 171, pp.00248.02016-pp.00248.02016.

Zhang, L., Zhou, W., Che, L., Rochaix, J.D., Lu, C., Li, W., and Peng, L. (2019). PPR Protein BFA2 Is Essential for the Accumulation of the atpH/F Transcript in Chloroplasts. Front Plant Sci 10, 446.

Zhang, L., Pu, H., Duan, Z., Li, Y., Liu, B., Zhang, Q., Li, W., Rochaix, J.-D., Liu, L., and Peng, L. (2018). Nucleus-Encoded Protein BFA1 Promotes Efficient Assembly of the Chloroplast ATP Synthase Coupling Factor 1. The Plant Cell 30, 1770-1788.

Zoschke, R., Kroeger, T., Belcher, S., Schöttler, M.A., Barkan, A., and SchmitzLinneweber, C. (2012). The pentatricopeptide repeat-SMR protein ATP4 promotes translation of the chloroplast atpB/E mRNA. The Plant journal : for cell and molecular biology $72,547-558$. 
970

\section{Supplemental tables}

Supplemental Table S1. AtCGL160 co-immunoprecipitation experiments. Differential enriched proteins in $P_{355}$ :AtCGL160 versus $P_{355}$ :AtCGL160C samples sorted by $\log _{2}$ fold change $\left(-\log _{10} P\right.$-value $\left.>1.5\right)$. Nucleus-encoded genes are written in capital letters.

\begin{tabular}{|c|c|c|c|}
\hline Protein IDs (Uniprot) & Gene names & $\log _{2} F C$ & $-\log _{10} p$-value \\
\hline 082279 & AtCGL160 & 6.495 & 4.644 \\
\hline P09468 & atpE & 6.38 & 2.301 \\
\hline Q42139 & ATPG & 5.231 & 4.747 \\
\hline P56760 & atpH & 4.886 & 3.176 \\
\hline Q9SSS9 & ATPD & 4.672 & 4.523 \\
\hline P19366 & $\operatorname{atp} B$ & 4.437 & 4.459 \\
\hline P56759 & atp $F$ & 4.399 & 5.913 \\
\hline P56757 & $\operatorname{atp} A$ & 4.235 & 6.41 \\
\hline Q01908 & ATPC1 & 4.156 & 4.772 \\
\hline Q2HIU0 & At3g15110 & 3.333 & 3.508 \\
\hline P56758 & atpl & 2.799 & 4.104 \\
\hline O49445 & LECRK72 & 2.346 & 3.115 \\
\hline Q67XC4 & $T B L 40$ & 2.268 & 2.402 \\
\hline Q8LCQ4 & LHCA6 & 2.082 & 3.71 \\
\hline A0A1P8B288, Q39099 & XTH4 & 1.983 & 4.126 \\
\hline Q41963 & TIP1-2 & 1.907 & 2.821 \\
\hline O22957 & At2g34040 & 1.632 & 2.829 \\
\hline $\begin{array}{l}\text { Q9SRL2, Q9M9X0, } \\
\text { F4J8G2, Q9SRL7, Q9S9U3 }\end{array}$ & $\begin{array}{l}\text { RLP32, RLP33, } \\
\text { RLP34, RLP35, RLP53 }\end{array}$ & 1.564 & 3.162 \\
\hline P38418, A0A1I9LPH1 & LOX2 & 1.544 & 2.047 \\
\hline Q8LBV4 & At1g78140 & 1.515 & 2.171 \\
\hline F4IUJ0, F4IUI9 & At2g26340 & 1.451 & 3.021 \\
\hline $\begin{array}{l}\text { Q9SF53, A0A1I9LSB4, } \\
\text { Q9M3D2 }\end{array}$ & RPL35A, RPL35C & 1.254 & 2.619 \\
\hline A0A1P8B6D0, Q9SUI4 & $P S A L$ & 1.193 & 2.938 \\
\hline Q9FFW9, F4KBJ3 & At5g38520 & 1.136 & 3.084 \\
\hline Q96242 & CYP74A & 1.078 & 2.371 \\
\hline Q9SYW8, F4K8I1 & Lhca2 & 0.941 & 2.356 \\
\hline Q9SR92 & STR10 & 0.839 & 2.913 \\
\hline P56777 & $p s b B$ & 0.807 & 2.072 \\
\hline Q9LHA6 & At3g28220 & 0.731 & 2.315 \\
\hline Q9S7N7 & PSAG & 0.575 & 2.157 \\
\hline
\end{tabular}


Supplemental Table S2. Primers used in this study.

\begin{tabular}{|c|c|c|}
\hline Primer name & Primer sequence 5' to $3^{\prime}$ & Comment \\
\hline oeAtCGL160_s & $\begin{array}{l}\text { GGGGACAAGTTTGTACAAAAAAGCAGG } \\
\text { CTCAATGGCGATTCTTAGTTACAT }\end{array}$ & Gateway primer \\
\hline oeAtCGL160_as & $\begin{array}{l}\text { GGGGACCACTTTGTACAAGAAAGCTGG } \\
\text { GTTTAATCACTGGCCTGTGTGT }\end{array}$ & Gateway primer \\
\hline oeAtCGL160C_s & $\begin{array}{l}\text { GGGGACAAGTTTGTACAAAAAAGCAGG } \\
\text { CTCAatgGAACAATATTTTAAGCTGAAAA }\end{array}$ & Gateway primer \\
\hline TP-GC9C_fus_s & $\begin{array}{l}\text { GGTCCACCGGAGTTGCTCCCGAACAAT } \\
\text { ATTTTAAGCTGAA }\end{array}$ & Fusion PCR \\
\hline TP-Gc9C_fus_as & $\begin{array}{l}\text { TTCAGCTTAAAATATTGTTCGGGAGCAA } \\
\text { CTCCGGTGGACC }\end{array}$ & Fusion PCR \\
\hline CGL160-MBP_s & AAAATCATTCTACCCAATAA & MBP cloning primer \\
\hline CGL160N-MBP_as & $\begin{array}{l}\text { GGTCCTGAATTCTTACCTGTCTTTAGCA } \\
\text { GCTTGTA }\end{array}$ & MBP cloning primer \\
\hline GST-CGL160N-s & $\begin{array}{l}\text { GGGGACAAGTTTGTACAAAAAAGCAGG } \\
\text { CTCAAAAATCATTCTACCCAATAAGAAA } \\
\text { CCTGA }\end{array}$ & Gateway primer \\
\hline GST-CGL160N-as & $\begin{array}{l}\text { GGGGACCACTTTGTACAAGAAAGCTGG } \\
\text { GTCTTACCTGTCTTTAGCAGCTTGTAC }\end{array}$ & Gateway primer \\
\hline cgl160cTP_probe_s & ATGGCGATTCTTAGTTACATCTCAGC & Northern-probe \\
\hline cgl160cTP_probe_as & GGGAGCAACTCCGGTG & Northern-probe \\
\hline pGBKT7-CGL160N_s & $\begin{array}{l}\text { GGTGGTCATATGAAAATCATTCTACCCA } \\
\text { ATAAGA }\end{array}$ & $\mathrm{Y} 2 \mathrm{H}$ cloning primer \\
\hline pGBKT7-CGL160N_as & $\begin{array}{l}\text { GGTCCTGAATTCTTACCTGTCTTTAGCA } \\
\text { GCTTGTA }\end{array}$ & $\mathrm{Y} 2 \mathrm{H}$ cloning primer \\
\hline pGADT7-alpha_s & $\begin{array}{l}\text { GGTGGTCATATGGTAACCATTAGAGCC } \\
\text { GACGA }\end{array}$ & $\mathrm{Y} 2 \mathrm{H}$ cloning primer \\
\hline pGADT7-alpha_as & $\begin{array}{l}\text { GGTCCTGAATTCTTATACTTTCTCCTGA } \\
\text { AGTA }\end{array}$ & $\mathrm{Y} 2 \mathrm{H}$ cloning primer \\
\hline pGADT7-beta_s & $\begin{array}{l}\text { GGTGGTCATATGAGAACAAATCCTACTA } \\
\text { CTTC }\end{array}$ & $\mathrm{Y} 2 \mathrm{H}$ cloning primer \\
\hline pGADT7-beta_as & $\begin{array}{l}\text { GGTCCTGAATTCTCATTTCTTCAATTTA } \\
\text { СTCT }\end{array}$ & $\mathrm{Y} 2 \mathrm{H}$ cloning primer \\
\hline pGADT7-gamma_s & $\begin{array}{l}\text { GGTGGTCATATGGCTTCCTCTGTTTCAC } \\
\text { CACT }\end{array}$ & $\mathrm{Y} 2 \mathrm{H}$ cloning primer \\
\hline pGADT7-gamma_as & $\begin{array}{l}\text { GGTCCTGAATTCTCAAACCTGTGCATTA } \\
\text { GCTC }\end{array}$ & $\mathrm{Y} 2 \mathrm{H}$ cloning primer \\
\hline pGADT7-delta_s & $\begin{array}{l}\text { GGTGGTCATATGGCCACCGCAGCATCA } \\
\text { AGCTA }\end{array}$ & Y2H cloning primer \\
\hline pGADT7-delta_as & $\begin{array}{l}\text { GGTCCTGAATTCTCAAGTAGCTAATTGA } \\
\text { ATCT }\end{array}$ & $\mathrm{Y} 2 \mathrm{H}$ cloning primer \\
\hline
\end{tabular}




\begin{tabular}{|c|c|c|}
\hline pGADT7-epsilon_s & $\begin{array}{l}\text { GGTGGTCATATGACCTTAAATCTTTGTG } \\
\text { TACTGACTC }\end{array}$ & $\mathrm{Y} 2 \mathrm{H}$ cloning primer \\
\hline pGADT7-epsilon_as & $\begin{array}{l}\text { GGTCCTGAATTCTCAAATCGTATTGAGA } \\
\text { GCCT }\end{array}$ & $\mathrm{Y} 2 \mathrm{H}$ cloning primer \\
\hline pGADT7-AtCGL160_s & $\begin{array}{l}\text { GGTGGTCATATGAAAATCATTCTACCCA } \\
\text { ATAAGA }\end{array}$ & $\mathrm{Y} 2 \mathrm{H}$ cloning primer \\
\hline pGADT7-AtCGL160_as & $\begin{array}{l}\text { GGTCCTGAATTCTTACCTGTCTTTAGCA } \\
\text { GCTTGTA }\end{array}$ & $\mathrm{Y} 2 \mathrm{H}$ cloning primer \\
\hline pGADT7-AtCGLD11_s & $\begin{array}{l}\text { GGTGGTCATATGTCTTCGAGTCTATGG } \\
\text { AAGCT }\end{array}$ & $\mathrm{Y} 2 \mathrm{H}$ cloning primer \\
\hline pGADT7-AtCGLD11_as & $\begin{array}{l}\text { GGTCCTGAATTCTTAACCCTGGAGTAAT } \\
\text { TTCA }\end{array}$ & $\mathrm{Y} 2 \mathrm{H}$ cloning primer \\
\hline pGADT7-atpFsoluble_s & $\begin{array}{l}\text { GGTGGTCATATGGATTTATTAGATAACC } \\
\text { GAAAG }\end{array}$ & $\mathrm{Y} 2 \mathrm{H}$ cloning primer \\
\hline pGADT7-atpFsoluble_as & $\begin{array}{l}\text { GGTCCTGAATTCTTAATCAGTTATTTCT } \\
\text { TTCATCG }\end{array}$ & $\mathrm{Y} 2 \mathrm{H}$ cloning primer \\
\hline pGADT7-atpGsoluble_s & $\begin{array}{l}\text { GGTGGTCATATGCCGCTTGGTAACTTC } \\
\text { ATGG }\end{array}$ & $\mathrm{Y} 2 \mathrm{H}$ cloning primer \\
\hline pGADT7-atpGsoluble_as & $\begin{array}{l}\text { GGTCCTGAATTCTTAAGAAGGAAGAAC } \\
\text { CTTCTTGAC }\end{array}$ & $\mathrm{Y} 2 \mathrm{H}$ cloning primer \\
\hline pGADT7_AtpBI-AD_s & CGCGAATTCATGAGAACAAATCCTAC & $\mathrm{Y} 2 \mathrm{H}$ cloning primer \\
\hline pGADT7_AtpBI-AD_as & $\begin{array}{l}\text { ACTCTCGAGTCAATTTCCCATATCAACC } \\
\text { AC }\end{array}$ & $\mathrm{Y} 2 \mathrm{H}$ cloning primer \\
\hline pGADT7_AtpBII-AD_s & ATGGAATTCCCTCTAAGTGTTCCAG & Y2H cloning primer \\
\hline pGADT7_AtpBII-AD_as & $\begin{array}{l}\text { AACCTCGAGTCAAGGTTGTAGCATAGT } \\
\text { TG }\end{array}$ & $\mathrm{Y} 2 \mathrm{H}$ cloning primer \\
\hline pGADT7_AtpBIII-AD_s & CTAGAATTCCGAATCGTTGGCGAG & $\mathrm{Y} 2 \mathrm{H}$ cloning primer \\
\hline pGADT7_AtpBIII-AD_as & GCGCTCGAGTCATTTCTTCAATTTACTC & $\mathrm{Y} 2 \mathrm{H}$ cloning primer \\
\hline pGBKT7_CGL160N_del29_74_s & GACTTAATCTGGAACAGAGATTTTATGG & Y2H cloning primer \\
\hline pGBKT7_CGL160N_del29_74_as & CATATGCAGGTCCTCCTCT & Y2H cloning primer \\
\hline pGBKT7_CGL160N_del75_105_s & GTCTTCTGGGTTTCTGAG & $\mathrm{Y} 2 \mathrm{H}$ cloning primer \\
\hline pGBKT7_CGL160N_del75_105_as & GTGGAAGTAATGGGATCTTC & $\mathrm{Y} 2 \mathrm{H}$ cloning primer \\
\hline pGBKT7_CGL160N_del106_134_s & CGTTGTGAAAAATCGTCTTGACAC & $\mathrm{Y} 2 \mathrm{H}$ cloning primer \\
\hline pGBKT7_CGL160N_del106_134_as & GACTTTTCCTTTGAAGGAGATGG & $\mathrm{Y} 2 \mathrm{H}$ cloning primer \\
\hline pGBKT7_CGL160N_del135_160_s & GAAGCTGGCACCTACACG & $\mathrm{Y} 2 \mathrm{H}$ cloning primer \\
\hline pGBKT7_CGL160N_del135_160_as & $\begin{array}{l}\text { CATTTAGAAGACGATGCAAGCTCTTTAC } \\
\text { TTAAATC }\end{array}$ & $\mathrm{Y} 2 \mathrm{H}$ cloning primer \\
\hline pGBKT7_CGL160N_del161_206_s & GAATTCCCGGGGATCCG & $\mathrm{Y} 2 \mathrm{H}$ cloning primer \\
\hline pGBKT7_CGL160N_del161_206_as & $\begin{array}{l}\text { CTATTTAGGAGACACAATAGCCTTACTC } \\
\text { ATTTG }\end{array}$ & $\mathrm{Y} 2 \mathrm{H}$ cloning primer \\
\hline
\end{tabular}




\section{Supplemental Figures}
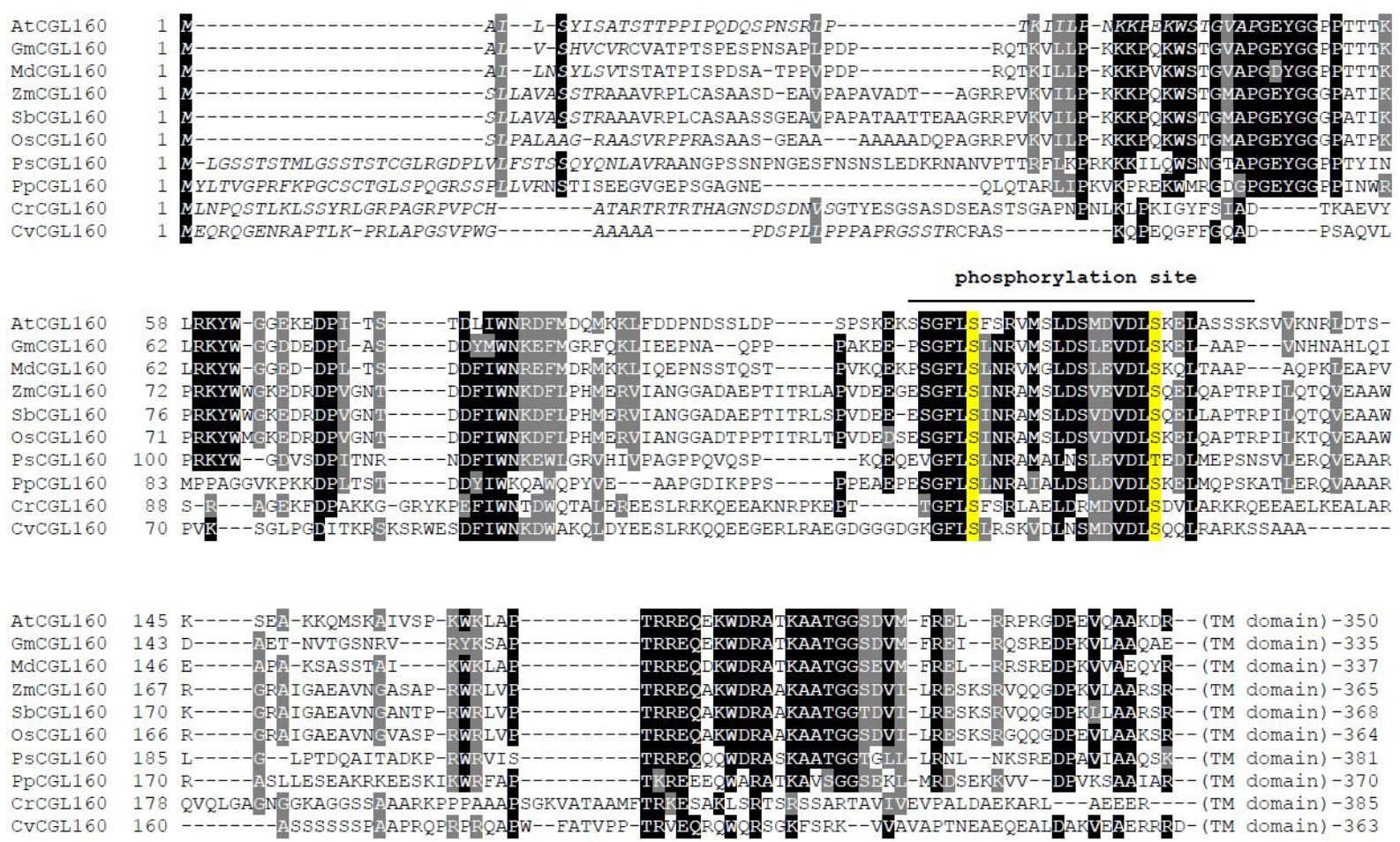

Supplemental Figure S1. Multiple alignment of the N-terminal portions of CGL160 sequences identified in species belonging to the green lineage. Chloroplast transit peptides predicted by ChloroP are depicted in italics. Similar and identical amino acids conserved in $70 \%$ of the sequences are highlighted in grey and black, respectively. The region that includes several identified phosphopeptides in AtCGL160 is indicated and two conserved $\mathrm{S} / \mathrm{T}$ residues are shown in yellow. Note that CGL160 transmembrane (TM) domains were omitted from the alignment. Sequence identifiers for CGL160 homologs are as follows: Arabidopsis thaliana (AtCGL160, NP_565711), Glycine max (GmCGL160, XP_006582279.1), Malus domestica (MdCGL160, XP_008353735.1), Zea mays (ZmCGL160, NP_001170362.2), Sorghum bicolor (SmCGL160, XP_021312638.1), Oryza sativa Japonica group (OsCGL160, XP_015619276.1), Picea sitchensis (PsCGL160, ABR16992.1), Physcomitrella patens (PpCGL160, XP_024381807.1), Chlamydomonas reinhardtii (CrCGL160, XP_001690237.1) and Chlorella variabilis (CvCGL160, XP_005844436.1). 
A

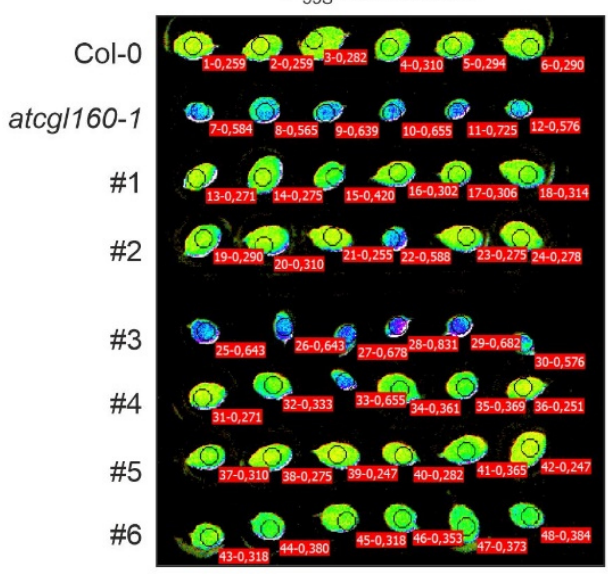

$P_{35 s}: A t C G L 160 N$

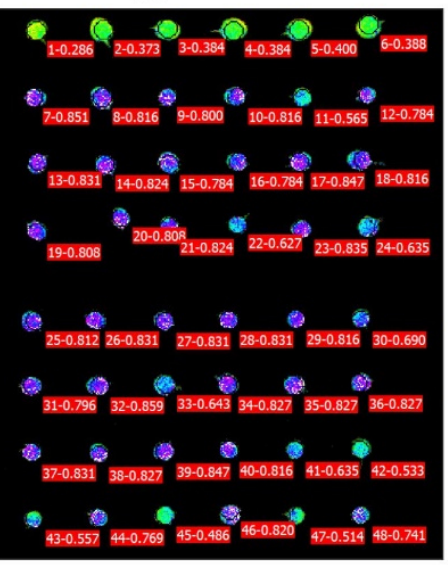

$P_{35}:$ AtCGL160C

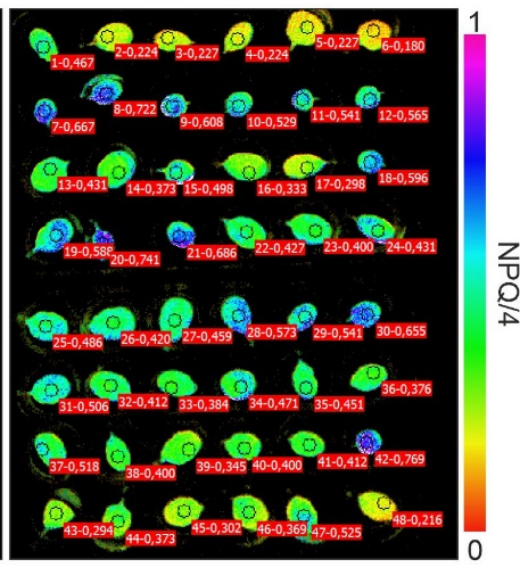

B
C

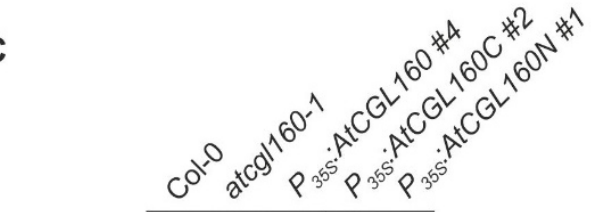

AtCGL160

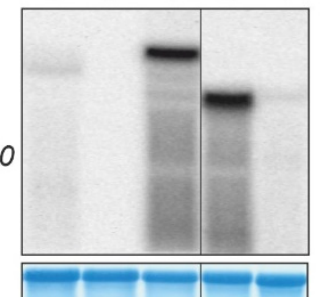

M.B.

\section{Supplemental Figure S2. Screening of $P_{35 s}:$ AtCGL160, $P_{355}$ :AtCGL160N and} $P_{35 s}$ :AtCGL160C plants. A, After transformation of atcgl160-1, T2 offspring of independent T1 plants (\#1-\#6) were examined using an Imaging-PAM (Walz, Effeltrich, Germany) system. Non-photochemical quenching (NPQ/4) was measured in light induction experiments on detached leaves after $8 \mathrm{~min}$ of irradiation at $100 \mu \mathrm{mol}$ photons $\mathrm{m}^{-2} \mathrm{~s}^{-1}$, and is indicated on a false-color scale from 0 to 1 . Col-0 and atcgl160-1 leaves served as controls. P35s:AtCGL160 lines \#1, \#2, \#4, \#5 and \#6 rescued the atcg/160-1 phenotype. Transformation of atcg/160-1 plants with the $P_{355}: A t C G L 160 N$ and $P_{355}:$ AtCGL160C constructs resulted in no complementation and partial complementation, respectively. B, AtCGL160 transcript levels in $P_{355}$ :AtCGL160C plants determined by Northern analysis. Note that RNA samples $(8 \mu \mathrm{g})$ of line \#2 were loaded twice for direct comparison of transcript levels with line \#1 $\left(P_{35 s}\right.$ :AtCGL160). C, Northern analyses of selected, homozygous lines (T3 generation). Total RNA $(20 \mu \mathrm{g})$ from 4-week-old Col-0, atcgl160-1, P35s:AtCGL160, P35s:AtCGL160N and $P_{355}$ :AtCGL $160 \mathrm{C}$ plants was size-fractionated on a denaturing formaldehyde gel and blotted onto a nylon membrane. Hybridization was carried out with a radioactive probe specific for the AtCGL160 chloroplast transit-peptide coding region. Line \#4 (P $P_{35 s}$ :AtCGL160) and line \#2 ( $P_{355}:$ AtCGL160C) were selected for further experiments due to their similar transcript levels. Methylene blue (M.B.) staining of the nylon membrane served as an RNA loading control in B and $\mathbf{C}$. 


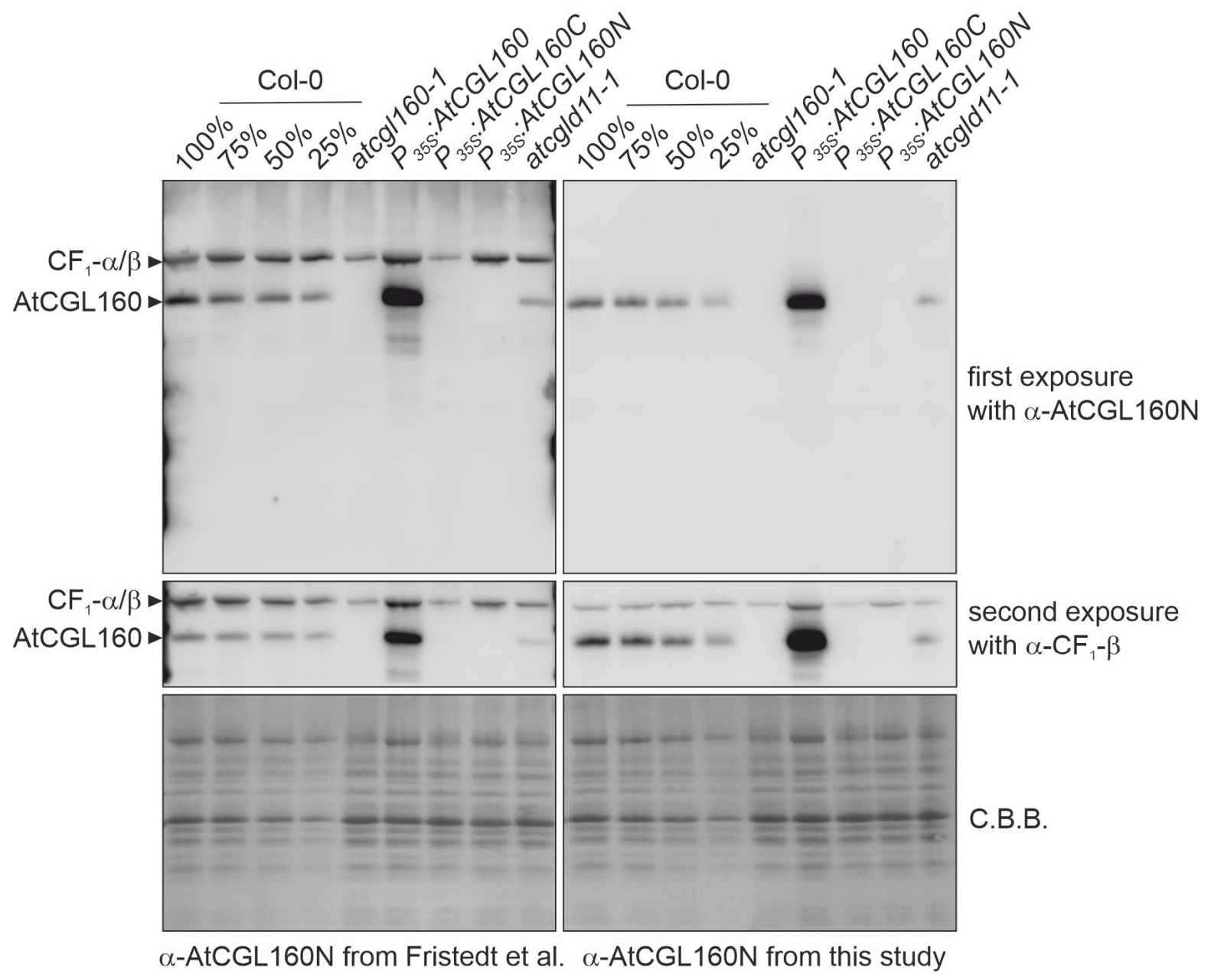

Supplemental Figure S3. Immunodetection of AtCGL160 in Col-0, atcgl160-1, $P_{35 s}:$ AtCGL160, $P_{35 s}$ :AtCGL160N, $P_{35 s}:$ AtCGL160C and atcgld11-1 plants. Thylakoid proteins were separated by denaturing SDS-PAGE and blotted onto PVDF membranes. Membranes were first probed with antibodies against AtCGL160N. After signal detection, membranes were re-probed with an antibody against $\mathrm{CF}_{1-}-\beta$. Coomassie brilliant blue staining (C.B.B.) of PVDF membranes is shown as a loading control. On the left, immunodetection analyses are shown for an AtCGL160 antibody (AS12 1853) which is commercially available from Agrisera and was employed in Fristedt et al. (2015). A side-by-side comparison with the newly generated antibody against the $\mathrm{N}$-terminal part of AtCGL160 is provided in the right panel. Note that antibody AS12 1853 from Agrisera binds nonspecifically to $C_{1}-\alpha$ or $C F_{1}-\beta$ and was therefore not considered for use in co-immunoprecipitation, cross-linking or 2D native/SDS-PAGE experiments. 
A

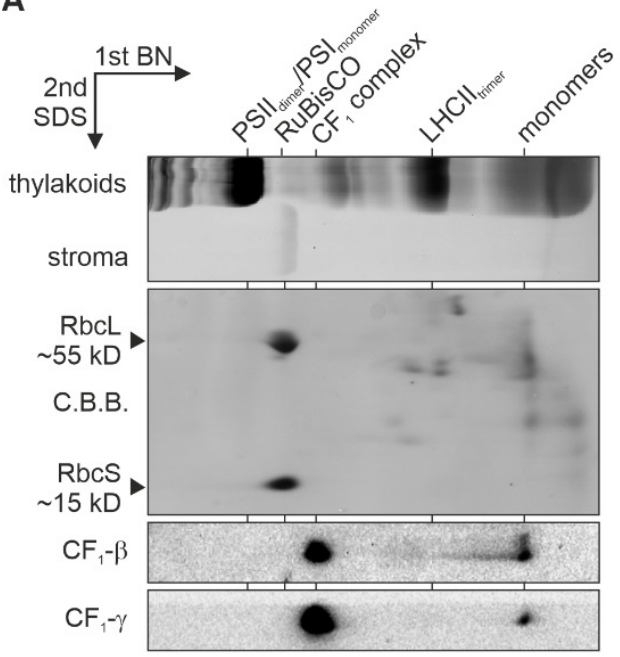

B

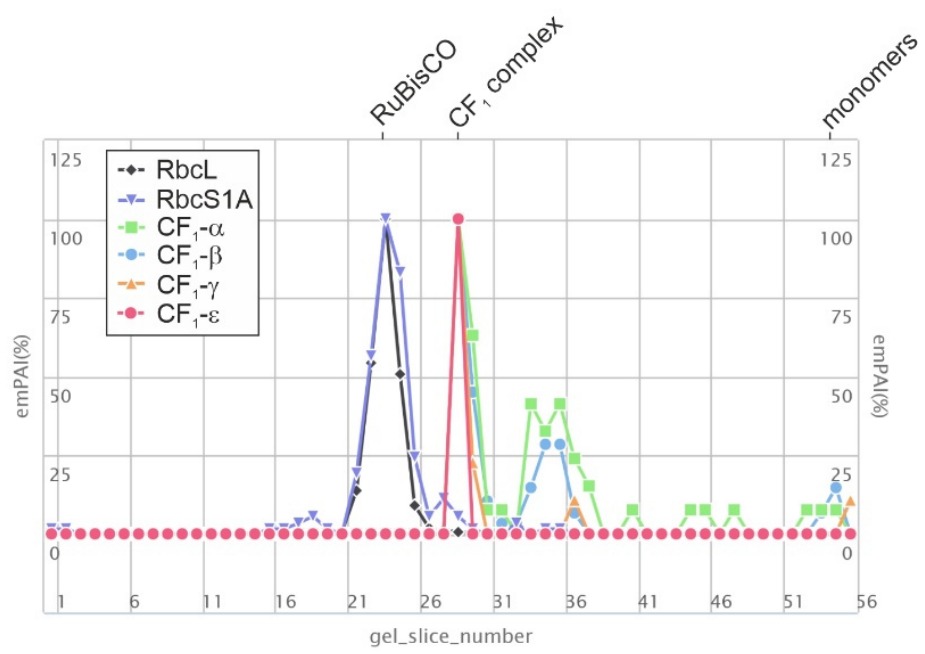

Supplemental Figure S4. Characterization of the stromal $\mathrm{CF}_{1}$ complex in atcgl160-1 plants. A, A stromal protein extract of atcg/160-1 plants was subjected to 2D gel electrophoresis (Blue Native- and SDS-PAGE) and immunodetection of $C_{1}-\beta$ and $C F_{1-\gamma}$. Coomassie brilliant blue (G-250) staining of the PVDF membrane after transfer visualized abundant stromal complexes such as RuBisCO, which is composed of RbcL and RbcS. Prominent thylakoid complexes of $P_{355}$ :AtCGL160C plants served as molecular mass standards. $\mathrm{B}$, Composition of the stromal $\mathrm{CF}_{1}$ sub-complex in Arabidopsis according to the Protein Co-migration Database for photosynthetic organisms (PCom-DB, http://pcomdb.lowtem.hokudai.ac.jp/proteins/top). Co-migration of RbcL (black diamonds) and RbcS (purple triangles) is provided for better comparison between PCom-DB results and the 2D gel analyses presented in panel A. Subunit content is quantified according to the exponentially modified protein abundance index (emPAl) method and is normalized for each individual subunit to the maximal emPAl identified in a gel slice (Ishihama et al., 2005). The maximal RuBisCO and $\mathrm{CF}_{1}$ content was detected in gel slices 24 and 29, respectively. Note that $\mathrm{CF}_{1}-\delta$ was not identified in the stromal $\mathrm{CF}_{1}$ subcomplex. 


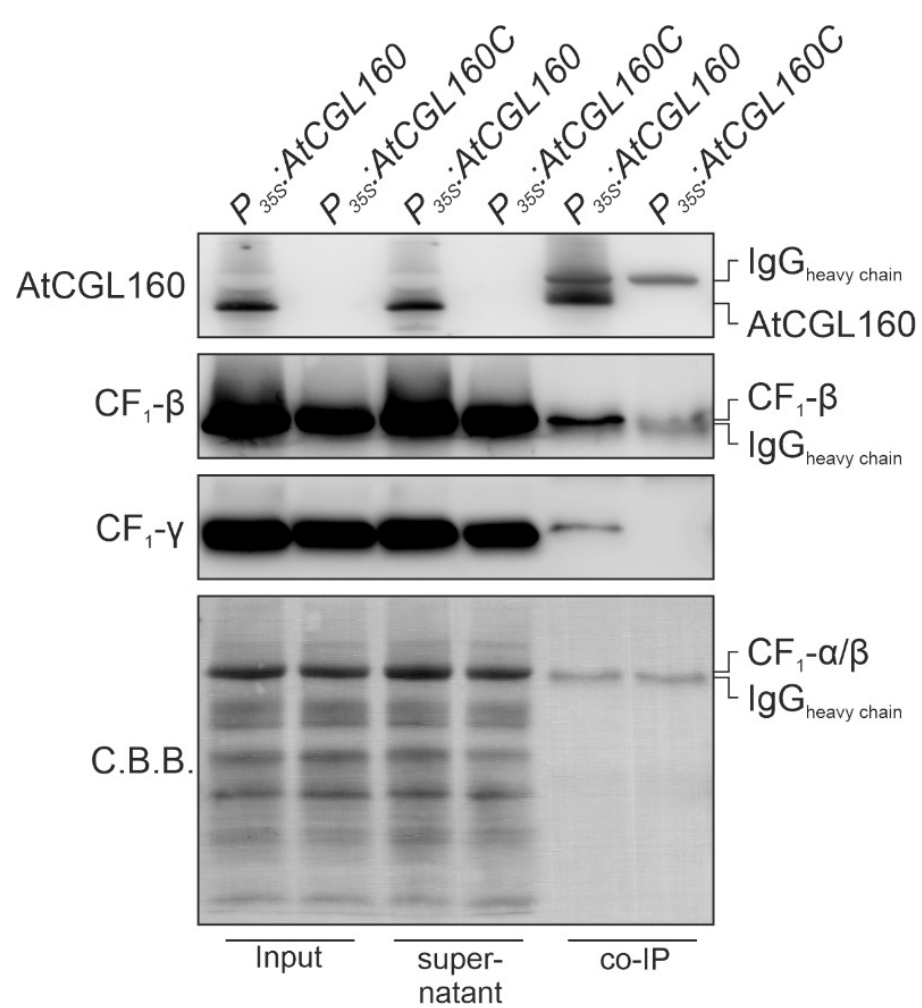

Supplemental Figure S5. Immunoblot analysis of AtCGL160 co-immunoprecipitation assays. A, Co-immunoprecipitation with NP40-solubilized thylakoids of oeAtCGL160 and oeAtCGL160C was repeated using reduced amounts of the AtCGL160 antibody. Protein Acoupled magnetic beads (Dynabeads, Thermo) coated with AtCGL160 antibody and coimmunoprecipated proteins (IP) were boiled in SDS loading buffer, separated by denaturing SDS-PAGE and blotted onto PVDF membranes. Samples of NP40-solubilized thylakoids before (Input) and after (Flow) incubation with AtCGL160 antibody were loaded as controls. Membranes were probed separately with antibodies against AtCGL160N and $C F_{1-\beta} \beta / \mathrm{CF}_{1-\gamma} \gamma$. The positions of the heavy chain of the AtCGL160 antibody are indicated (IgG). Coomassie brilliant blue staining (C.B.B.) is shown as loading control, and the positions of $\mathrm{CF}_{1-\alpha} \alpha / \beta$ are indicated. 

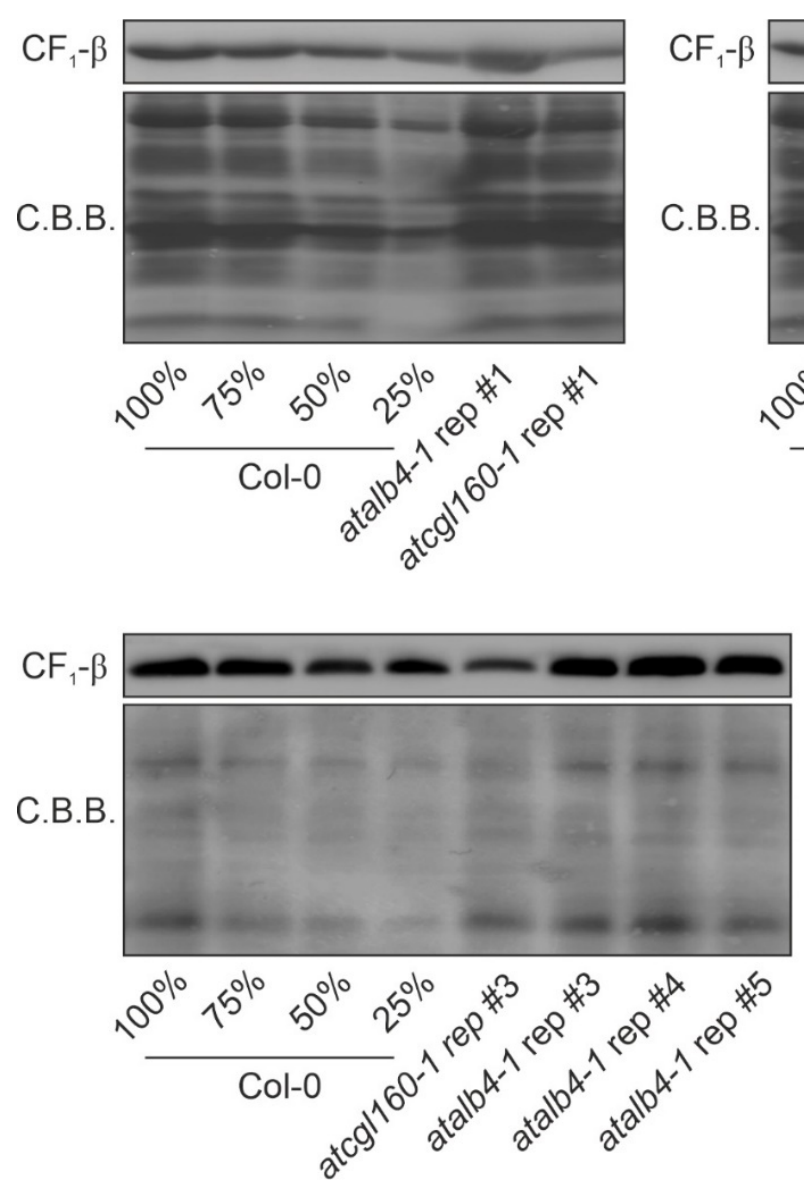
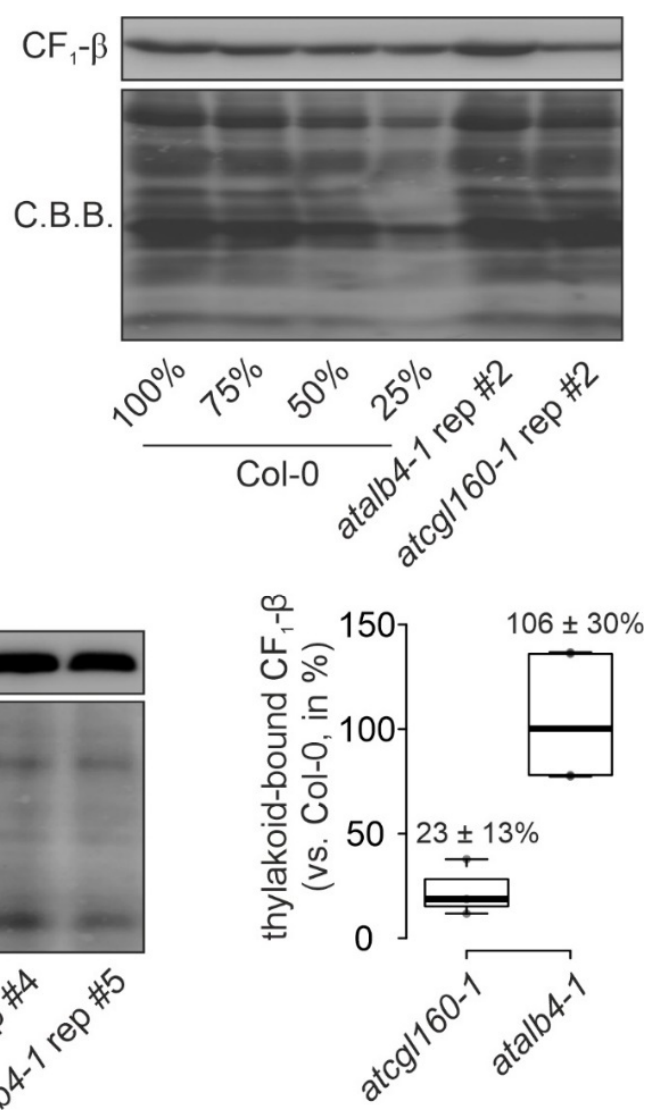

\begin{abstract}
Supplemental Figure S6. Quantification of thylakoid-bound $\mathrm{CF}_{1-\beta} \boldsymbol{\beta}$ subunits in atalb4-1 Arabidopsis mutant lines. Thylakoid proteins were isolated from Col-0, atcg/160-1 and atalb4-1 (SALK_136199C) plants grown under short-day conditions, fractionated on SDSPAGE, transferred to PVDF membranes and probed with $\mathrm{CF}_{1-}-\beta$ antibodies. Membranes were stained with Coomassie brilliant blue G-250 (C.B.B.) as loading control. Signals were quantified relative to signals detected in the wild-type sample using the Bio-1D software (version 15.03, Vilber Lourmat, Eberhardzell, Germany) and are provided as percentages. Horizontal lines represent the median, and boxes indicate the 25th and 75th percentiles. Whiskers extend the interquartile range by a factor of $1.5 \times$. Means \pm standard deviations are provided above the boxes. Quantification is based on three and five replicates (rep) for atcgl160-1 and atalb4-1 samples, respectively.
\end{abstract}

\title{
Additional references in Supplemental Figures
}

Ishihama, Y., Oda, Y., Tabata, T., Sato, T., Nagasu, T., Rappsilber, J., and Mann, M. (2005). Exponentially modified protein abundance index (emPAl) for estimation of absolute protein amount in proteomics by the number of sequenced peptides per protein. Mol Cell Proteomics 4, 1265-1272. 